\title{
SUPPLEMENT TO "ESTIMATION AND MODEL SELECTION IN GENERALIZED ADDITIVE PARTIAL LINEAR MODELS FOR CORRELATED DATA WITH DIVERGING NUMBER OF COVARIATES"
}

\author{
By Li Wang, Lan Xue, Annie Qu, and Hua liang \\ University of Georgia, Oregon State University, University of Illinois at \\ Urbana-Champaign, and George Washington University
}

In this document, we collect a number of technical lemmas and their proofs. The technical lemmas are used in the proofs of Theorems 1-5 in the paper.

S.1. Preliminary lemmas. Let $\boldsymbol{B}_{l}=\left(B_{l, 1}, \ldots, B_{l, J_{n}}\right)^{\mathrm{T}}$ be a set of orthonormal bases of $\varphi_{l}^{0, n}$ with respect to inner product as defined in (A.1) of the main text, for $l=1, \ldots, d_{x}$. Let $\boldsymbol{B}=\left(\boldsymbol{B}_{1}^{\mathrm{T}}, \ldots, \boldsymbol{B}_{d_{x}}^{\mathrm{T}}\right)^{\mathrm{T}}$, then $\boldsymbol{B}$ is a set of bases for $\mathcal{M}_{n}$. In the following, let $\boldsymbol{a}$ be a unit $d_{n}$-vector unless otherwise specified. Recall $\rho_{n}=((1-\delta) / 2)^{\left(d_{x}-1\right) / 2}$.

Lemma S.1. Under condition (C1), there exists a constant $c>0$, such that

$$
\|\alpha\|^{2} \geq c \rho_{n}^{2}\left(\alpha_{0}^{2}+\sum_{l=1}^{d_{x}}\left\|\alpha_{l}\right\|^{2}\right), \forall \alpha=\alpha_{0}+\sum_{l=1}^{d_{x}} \alpha_{l} \in \mathcal{M} .
$$

Proof. The result follows from an extension of Lemma 3 in [3].

Lemma S.2. Under Conditions (C2), (C3) and (C8), there exist constants $C \geq c>0$, such that, as $n \rightarrow \infty$,

$$
c \rho_{n}^{2} \leq E\left\{\boldsymbol{a}^{\mathrm{T}}\left(n^{-1} \sum_{i=1}^{n} \boldsymbol{D}_{i}^{\mathrm{T}} \boldsymbol{D}_{i}\right) \boldsymbol{a}\right\} \leq C
$$

and

$$
c \rho_{n}^{2} \leq \boldsymbol{a}^{\mathrm{T}}\left(n^{-1} \sum_{i=1}^{n} \boldsymbol{D}_{i}^{\mathrm{T}} \boldsymbol{D}_{i}\right) \boldsymbol{a} \leq C,
$$

except in an event whose probability tends to zero. 
The proof of Lemma S.2 is analogous to that of Lemma A.3 given by [4], replacing $\boldsymbol{D}_{i}$ by $\boldsymbol{B}_{i}$ and applying Lemma S.1, thus omitted.

In the following, define a $d_{n}$-vector $\boldsymbol{G}_{n, k}(\boldsymbol{\theta})=\frac{1}{n} \sum_{i=1}^{n} \boldsymbol{g}_{i k}(\boldsymbol{\theta})$, for $k=$ $1, \ldots, K$, where

$$
\boldsymbol{g}_{i k}(\boldsymbol{\theta})=\boldsymbol{g}_{i k}\left(\boldsymbol{\mu}_{i}(\boldsymbol{\theta})\right)=\boldsymbol{D}_{i}^{\mathrm{T}} \boldsymbol{\Delta}_{i} \boldsymbol{A}_{i}^{-1 / 2} \boldsymbol{M}_{k} \boldsymbol{A}_{i}^{-1 / 2}\left\{\boldsymbol{Y}_{i}-\boldsymbol{\mu}_{i}(\boldsymbol{\theta})\right\} .
$$

Then $\boldsymbol{G}_{n}$ in $(2.3)$ of the main text can be written as $\left(\boldsymbol{G}_{n, 1}^{\mathrm{T}}(\boldsymbol{\theta}), \ldots, \boldsymbol{G}_{n, K}^{\mathrm{T}}(\boldsymbol{\theta})\right)^{\mathrm{T}}$. Note that the regression function $\boldsymbol{\eta}_{i}(\boldsymbol{\theta}) \equiv \boldsymbol{D}_{i}^{\mathrm{T}} \boldsymbol{\theta}$ can be decomposed as $\boldsymbol{\eta}_{i}(\boldsymbol{\theta})=\boldsymbol{\eta}_{0, i}+\boldsymbol{\zeta}_{i}(\boldsymbol{\theta})$, where

$$
\begin{aligned}
\boldsymbol{\zeta}_{i}(\boldsymbol{\theta}) & =\left(\alpha_{0}-\widetilde{\alpha}\right)\left(\boldsymbol{X}_{i}\right)+\boldsymbol{B}_{i}(\widetilde{\boldsymbol{\gamma}}-\boldsymbol{\gamma})+\boldsymbol{Z}_{i}\left(\boldsymbol{\beta}_{0}-\boldsymbol{\beta}\right) \\
& \equiv \boldsymbol{\zeta}_{i 1}(\alpha)+\boldsymbol{\zeta}_{i 2}(\boldsymbol{\gamma})+\boldsymbol{\zeta}_{i 3}(\boldsymbol{\beta}) .
\end{aligned}
$$

Thus,

$$
\boldsymbol{\mu}_{i}(\boldsymbol{\theta})=\mu\left\{\boldsymbol{\eta}_{0, i}+\boldsymbol{\zeta}_{i}(\boldsymbol{\theta})\right\} .
$$

Similar to (S.1), we express

$$
\begin{aligned}
g_{i k}^{0}(\boldsymbol{\theta}) & =\boldsymbol{D}_{i}^{\mathrm{T}} \boldsymbol{\Delta}_{0, i}^{\mathrm{T}} \boldsymbol{V}_{0, i}^{(k)}\left[\boldsymbol{e}_{i}+\boldsymbol{\Delta}_{0, i}\left\{\boldsymbol{\zeta}_{i 1}(\alpha)+\boldsymbol{\zeta}_{i 2}(\boldsymbol{\gamma})+\boldsymbol{\zeta}_{i 3}(\boldsymbol{\beta})\right\}\right] \\
& \equiv g_{i k 1}^{0}+g_{i k 2}^{0}(\alpha)+g_{i k 3}^{0}(\boldsymbol{\gamma})+g_{i k 4}^{0}(\boldsymbol{\beta})
\end{aligned}
$$

where

$$
\begin{aligned}
& g_{i k 1}^{0}=\boldsymbol{D}_{i}^{\mathrm{T}} \boldsymbol{\Delta}_{0, i} \boldsymbol{V}_{0, i}^{(k)} \boldsymbol{e}_{i}, \quad g_{i k 2}^{0}(\alpha)=\boldsymbol{D}_{i}^{\mathrm{T}} \boldsymbol{\Gamma}_{0, i}^{(k)} \boldsymbol{\zeta}_{i 1}(\alpha) \\
& g_{i k 3}^{0}(\boldsymbol{\gamma})=\boldsymbol{D}_{i}^{\mathrm{T}} \boldsymbol{\Gamma}_{0, i}^{(k)} \boldsymbol{\zeta}_{i 2}(\boldsymbol{\gamma}), \quad g_{i k 4}^{0}(\boldsymbol{\beta})=\boldsymbol{D}_{i}^{\mathrm{T}} \boldsymbol{\Gamma}_{0, i}^{(k)} \boldsymbol{\zeta}_{i 3}(\boldsymbol{\beta})
\end{aligned}
$$

Let $g_{k 1}^{0}=\frac{1}{n} \sum_{i=1}^{n} g_{i k 1}^{0}$, and define $g_{k 2}^{0}(\alpha), g_{k 3}^{0}(\boldsymbol{\gamma}), g_{k 4}^{0}(\boldsymbol{\beta})$ similarly. Next define $\boldsymbol{G}_{n, k}^{0}(\boldsymbol{\theta})=\frac{1}{n} \sum_{i=1}^{n} \boldsymbol{g}_{i k}^{0}(\boldsymbol{\theta})$, then define $\boldsymbol{G}_{n}^{0}(\boldsymbol{\theta}), \boldsymbol{C}_{n}^{0}(\boldsymbol{\theta}), Q_{n}^{0}(\boldsymbol{\theta})$ similarly as $\boldsymbol{G}_{n}(\boldsymbol{\theta}), \boldsymbol{C}_{n}(\boldsymbol{\theta}), Q_{n}(\boldsymbol{\theta})$ but replacing $\boldsymbol{g}_{i k}$ with $\boldsymbol{g}_{i k}^{0}$. Furthermore, let $\Theta_{n}\left(C_{1}, C_{2}\right)=\left\{\boldsymbol{\theta}=\left(\boldsymbol{\beta}^{\mathrm{T}}, \boldsymbol{\gamma}^{\mathrm{T}}\right)^{\mathrm{T}}:\left\|\boldsymbol{\beta}-\boldsymbol{\beta}_{0}\right\|=C_{1} n^{-1 / 2} d_{n}^{1 / 2},\left\|\boldsymbol{B}^{\mathrm{T}}(\boldsymbol{\gamma}-\widetilde{\boldsymbol{\gamma}})\right\|_{n}=\right.$ $\left.C_{2} n^{-1 / 2} d_{n}^{1 / 2}\right\}$ for positive constants $C_{1}$ and $C_{2}$.

LemmA S.3. Under the conditions of Theorem 1, $\left\|g_{k 1}^{0}\right\|=O_{P}\left(n^{-1 / 2}\right)$, $\left\|g_{k 2}^{0}(\alpha)\right\|=O_{P}\left(J_{n}^{-r} d_{x}^{1 / 2}\right)$. Furthermore, there exist constants $0<c_{2} \leq c_{1}$, $0<c_{4} \leq c_{3}$ such that for any $\boldsymbol{\theta} \in \Theta_{n}\left(C_{1}, C_{2}\right)$,

$$
\begin{aligned}
& c_{2} C_{1} \rho_{n} n^{-1 / 2} d_{n}^{1 / 2} \leq\left\|g_{k 3}^{0}(\boldsymbol{\gamma})\right\| \leq c_{1} C_{1} n^{-1 / 2} d_{n}^{1 / 2}, \\
& c_{4} C_{2} \rho_{n} n^{-1 / 2} d_{n}^{1 / 2} \leq\left\|g_{k 4}^{0}(\boldsymbol{\beta})\right\| \leq c_{3} C_{2} n^{-1 / 2} d_{n}^{1 / 2},
\end{aligned}
$$

except in an event whose probability goes to 0 as $n \rightarrow \infty$. 
Proof. For any $k=1, \ldots, K, \boldsymbol{a}^{\mathrm{T}} g_{k 1}^{0}$ has mean 0 , and by Conditions (C5), (C6) and Lemma S.2, there exists a constant $c>0$, such that

$$
\operatorname{Var}\left(\boldsymbol{a}^{\mathrm{T}} g_{k 1}^{0}\right)=\frac{1}{n} E \boldsymbol{a}^{\mathrm{T}} g_{i k 1}^{0} g_{i k 1}^{0 \mathrm{~T}} \boldsymbol{a} \leq \frac{c}{n} \boldsymbol{a}^{\mathrm{T}} E\left(\boldsymbol{D}_{i}^{\mathrm{T}} \boldsymbol{D}_{i}\right) \boldsymbol{a} \leq \frac{c}{n} \boldsymbol{a}^{\mathrm{T}} \boldsymbol{a}=O\left(n^{-1}\right) .
$$

Therefore $\left\|g_{k 1}^{0}\right\|=\sup _{\boldsymbol{a}}\left|\boldsymbol{a}^{\mathrm{T}} g_{k 1}^{0}\right|=O_{P}\left(n^{-1 / 2}\right)$. The rest of the results follow similarly from Lemma S.2 and Lemma B.8 of the Supplement of [5].

Lemma S.4. Under Conditions (C1)-(C8), there exist constants $0<$ $c_{6} \leq c_{5}, 0<c_{8} \leq c_{7}, 0<c_{10} \leq c_{9}$ such that for any $\boldsymbol{\theta} \in \Theta_{n}\left(C_{1}, C_{2}\right)$,

$$
\begin{gathered}
c_{6}\left(C_{1}+C_{2}\right) n^{-1 / 2} d_{n}^{1 / 2} \rho_{n} \leq\left\|\boldsymbol{G}_{n}^{0}(\boldsymbol{\theta})\right\| \leq c_{5}\left(C_{1}+C_{2}\right) n^{-1 / 2} d_{n}^{1 / 2}, \\
c_{8} \rho_{n} \leq \lambda_{\min }\left\{\boldsymbol{C}_{n}^{0}(\boldsymbol{\theta})\right\}<\lambda_{\max }\left\{\boldsymbol{C}_{n}^{0}(\boldsymbol{\theta})\right\} \leq c_{7}, \\
c_{10}\left(C_{1}+C_{2}\right) n^{-1} d_{n} \leq Q_{n}^{0}(\boldsymbol{\theta}) \leq c_{9}\left(C_{1}+C_{2}\right) \rho_{n}^{-1} n^{-1} d_{n},
\end{gathered}
$$

except in an event whose probability goes to 0 as $n \rightarrow \infty$.

Proof. Equation (S.4) follows immediately from Lemma S.3. Next let $\widetilde{\boldsymbol{D}}_{i}=$ $\boldsymbol{I}_{K} \otimes \boldsymbol{D}_{i}$, where $\boldsymbol{I}_{K}$ is the identity matrix of order $K$, and $\otimes$ denotes the Kronecker product. Furthermore, let $\boldsymbol{M}_{\text {diag }}=\operatorname{diag}\left(\boldsymbol{M}_{1}, \ldots, \boldsymbol{M}_{K}\right)$. To show (S.5), Conditions (C5) and (C6) ensure that it is sufficient to show that there exist constants $c^{*}$ and $C_{*}$ such that when $n$ is large enough,

$$
c^{*} \rho_{n} \leq \frac{1}{n} \sum_{i=1}^{n} \boldsymbol{a}^{\mathrm{T}} \widetilde{\boldsymbol{D}}_{i}^{\mathrm{T}} \boldsymbol{M}_{\mathrm{diag}} \boldsymbol{M}_{\mathrm{diag}}^{\mathrm{T}} \widetilde{\boldsymbol{D}}_{i} \boldsymbol{a} \leq C^{*},
$$

for any $\boldsymbol{a}=\left(\boldsymbol{a}_{0}^{\mathrm{T}}, \ldots, \boldsymbol{a}_{K}^{\mathrm{T}}\right)^{\mathrm{T}}$ with each $\boldsymbol{a}_{k} \in \boldsymbol{R}^{d_{n}}$ and $\boldsymbol{a}^{\mathrm{T}} \boldsymbol{a}=1$. The eigenvalues of $\boldsymbol{M}_{\text {diag }} \boldsymbol{M}_{\text {diag }}^{\mathrm{T}}$ are the squares of the singular values of $\boldsymbol{M}_{\text {diag }}$, which are bounded from 0 and $+\infty$ by Condition (C7). Then

$$
\frac{1}{n} \sum_{i=1}^{n} \boldsymbol{a}^{\mathrm{T}} \widetilde{\boldsymbol{D}}_{i}^{\mathrm{T}} \boldsymbol{M}_{\mathrm{diag}} \boldsymbol{M}_{\mathrm{diag}}^{\mathrm{T}} \widetilde{\boldsymbol{D}}_{i} \boldsymbol{a} \asymp \frac{1}{n} \sum_{i=1}^{n} \boldsymbol{a}^{\mathrm{T}} \widetilde{\boldsymbol{D}}_{i}^{\mathrm{T}} \widetilde{\boldsymbol{D}}_{i} \boldsymbol{a} .
$$

By Lemma S.2,

$$
\begin{aligned}
c \rho_{n} & \leq C \sum_{k=1}^{K} \boldsymbol{a}_{k}^{\mathrm{T}} \boldsymbol{a}_{k} \leq \frac{1}{n} \sum_{i=1}^{n} \boldsymbol{a}^{\mathrm{T}} \widetilde{\boldsymbol{D}}_{i}^{\mathrm{T}} \widetilde{\boldsymbol{D}}_{i} \boldsymbol{a}=\sum_{k=1}^{K} \boldsymbol{a}_{k}^{\mathrm{T}} \frac{1}{n} \sum_{i=1}^{n} \boldsymbol{D}_{i}^{\mathrm{T}} \boldsymbol{D}_{i} \boldsymbol{a}_{k} \\
& \leq C \sum_{k=1}^{K} \boldsymbol{a}_{k}^{\mathrm{T}} \boldsymbol{a}_{k}=C .
\end{aligned}
$$


Therefore (S.7) holds. Observing that

$$
\begin{gathered}
\lambda_{\max }^{-1}\left\{\boldsymbol{C}_{n}^{0}(\boldsymbol{\theta})\right\}\left\|\boldsymbol{G}_{n}^{0}(\boldsymbol{\theta})\right\|^{2} \leq Q_{n}^{0}(\boldsymbol{\theta})=\boldsymbol{G}_{n}^{0 \mathrm{~T}}(\boldsymbol{\theta})\left(\boldsymbol{C}_{n}^{0}(\boldsymbol{\theta})\right)^{-1} \boldsymbol{G}_{n}^{0}(\boldsymbol{\theta}) \\
\leq \lambda_{\min }^{-1}\left\{\boldsymbol{C}_{n}^{0}(\boldsymbol{\theta})\right\}\left\|\boldsymbol{G}_{n}^{0}(\boldsymbol{\theta})\right\|^{2}
\end{gathered}
$$

thus (S.6) follows from Lemma S.2 and (S.4).

Lemma S.5. Under Conditions (C1)-(C8), for some $C_{1}$ and $C_{2}$ sufficiently large,

$$
\begin{gathered}
\sup _{\boldsymbol{\theta} \in \Theta_{n}\left(C_{1}, C_{2}\right)}\left\|\boldsymbol{G}_{n}(\boldsymbol{\theta})-\boldsymbol{G}_{n}^{0}(\boldsymbol{\theta})\right\| \\
=O_{P}\left(J_{n}^{-2 r} d_{n}^{1 / 2} d_{x}+n^{-1} d_{n}^{3 / 2}+n^{-1 / 2} J_{n}^{-r} d_{n} d_{x}^{1 / 2}\right), \\
\sup _{\boldsymbol{\theta} \in \Theta_{n}\left(C_{1}, C_{2}\right)}\left\|\boldsymbol{C}_{n}(\boldsymbol{\theta})-\boldsymbol{C}_{n}^{0}(\boldsymbol{\theta})\right\|=O_{P}\left(n^{-1 / 2} d_{n}\right),
\end{gathered}
$$

$(\mathrm{S} .10) \sup _{\boldsymbol{\theta} \in \Theta_{n}\left(C_{1}, C_{2}\right)}\left|Q_{n}(\boldsymbol{\theta})-Q_{n}^{0}(\boldsymbol{\theta})\right|$

$$
=O_{P}\left\{\rho_{n}^{-1}\left(n J_{n}^{-4 r} d_{n} d_{x}^{2}+\rho_{n}^{-1} n^{-1 / 2} d_{n}^{3}+J_{n}^{-2 r} d_{n}^{2} d_{x}\right)\right\} .
$$

Proof. Similar to [5], it is sufficient to show that (S.8) holds for each of its components $\boldsymbol{G}_{n, k}(\boldsymbol{\theta})-\boldsymbol{G}_{n, k}^{0}(\boldsymbol{\theta})$ with $k=1, \ldots, K$. The Taylor expansion of $\boldsymbol{a}^{\mathrm{T}} \boldsymbol{G}_{n, k}(\boldsymbol{\theta}),(\mathrm{S} .2)$ and (S.3) implies that

$$
\begin{aligned}
\boldsymbol{a}^{\mathrm{T}} \boldsymbol{G}_{n, k}(\boldsymbol{\theta})=\boldsymbol{a}^{\mathrm{T}} \boldsymbol{G}_{n, k}(\boldsymbol{\mu}(\boldsymbol{\theta})) \\
=\frac{1}{n} \sum_{i=1}^{n} \boldsymbol{a}^{\mathrm{T}} \boldsymbol{D}_{i}^{\mathrm{T}} \boldsymbol{\Delta}_{i}\left\{\boldsymbol{\mu}_{i}\left(\boldsymbol{\eta}_{0, i}+\boldsymbol{\zeta}_{i}(\boldsymbol{\theta})\right)\right\} \boldsymbol{A}_{i}^{-1 / 2}\left\{\boldsymbol{\mu}_{i}\left(\boldsymbol{\eta}_{0, i}+\boldsymbol{\zeta}_{i}(\boldsymbol{\theta})\right)\right\} \\
\quad \times \boldsymbol{M}_{k} \boldsymbol{A}_{i}^{-1 / 2}\left\{\boldsymbol{\mu}_{i}\left(\boldsymbol{\eta}_{0, i}+\boldsymbol{\zeta}_{i}(\boldsymbol{\theta})\right)\right\}\left\{\boldsymbol{y}_{i}-\boldsymbol{\mu}_{i}\left(\boldsymbol{\eta}_{0, i}+\boldsymbol{\zeta}_{i}(\boldsymbol{\theta})\right)\right\} \\
=\frac{1}{n} \sum_{i=1}^{n} \boldsymbol{a}^{\mathrm{T}} \boldsymbol{D}_{i}^{\mathrm{T}} \boldsymbol{\Delta}_{0, i} \boldsymbol{V}_{0, i}^{(k)} \boldsymbol{e}_{i}+\frac{1}{n} \sum_{i=1}^{n} \boldsymbol{a}^{\mathrm{T}} \boldsymbol{D}_{i}^{\mathrm{T}} \boldsymbol{\Gamma}_{0, i}^{(k)} \boldsymbol{\zeta}_{i}(\boldsymbol{\theta}) \\
\quad+\frac{1}{n} \sum_{i=1}^{n} \boldsymbol{\zeta}_{i}^{\mathrm{T}}(\boldsymbol{\theta}) \boldsymbol{\Delta}_{0, i} \frac{\partial \boldsymbol{a}^{\mathrm{T}} \boldsymbol{D}_{i}^{\mathrm{T}} \boldsymbol{\Delta}_{0, i} \boldsymbol{A}_{0, i}^{-1 / 2} \boldsymbol{M}_{k} \boldsymbol{A}_{0, i}^{-1 / 2}}{\partial \boldsymbol{\mu}_{i}} \boldsymbol{e}_{i}+R_{n}^{*}\left(\boldsymbol{\mu}^{*}\right),
\end{aligned}
$$

where $R_{n}^{*}\left(\boldsymbol{\mu}^{*}\right)=\frac{1}{n} \sum_{i=1}^{n} R_{n i}^{*}\left(\boldsymbol{\mu}_{i}^{*}\right)$ with

$$
R_{n i}^{*}\left(\boldsymbol{\mu}_{i}^{*}\right)=\frac{1}{2} \boldsymbol{\zeta}_{i}^{\mathrm{T}} \boldsymbol{\Delta}_{i} \frac{\partial^{2} \boldsymbol{a}^{\mathrm{T}} \boldsymbol{D}_{i}^{\mathrm{T}} \boldsymbol{\Delta}_{i}\left(\boldsymbol{\mu}_{i}\right) \boldsymbol{A}_{i}^{-1 / 2}\left(\boldsymbol{\mu}_{i}\right) \boldsymbol{M}_{k} \boldsymbol{A}_{i}^{-1 / 2}\left(\boldsymbol{\mu}_{i}\right)\left(\boldsymbol{y}_{i}-\boldsymbol{\mu}_{i}\right)}{\partial \boldsymbol{\mu}_{i} \partial \boldsymbol{\mu}_{i}^{\mathrm{T}}} \boldsymbol{\Delta}_{i} \boldsymbol{\zeta}_{i}
$$


evaluated at $\boldsymbol{\mu}_{i}^{*}=g^{-1}\left(\boldsymbol{\eta}_{0, i}+\tau_{i} \boldsymbol{\zeta}_{i}\right)$ with $0<\tau_{i}<1$. Then one has

$$
\begin{aligned}
\boldsymbol{a}^{\mathrm{T}}\left\{\boldsymbol{G}_{n, k}(\boldsymbol{\theta})-\boldsymbol{G}_{n, k}^{0}(\boldsymbol{\theta})\right\} & =\frac{1}{n} \sum_{i=1}^{n} \boldsymbol{\zeta}_{i}^{\mathrm{T}}(\boldsymbol{\theta}) \boldsymbol{\Delta}_{0, i} \frac{\partial \boldsymbol{a}^{\mathrm{T}} \boldsymbol{D}_{i}^{\mathrm{T}} \boldsymbol{\Delta}_{0, i} \boldsymbol{A}_{0, i}^{-1 / 2} \boldsymbol{M}_{k} \boldsymbol{A}_{0, i}^{-1 / 2}}{\partial \boldsymbol{\mu}_{i}} \boldsymbol{e}_{i} \\
+ & R_{n}^{*}\left(\boldsymbol{\mu}^{*}\right)=I_{k}(\boldsymbol{\theta})+R_{n}^{*}\left(\boldsymbol{\mu}^{*}\right) .
\end{aligned}
$$

Let

$$
\boldsymbol{L}_{i}^{(k)}=\boldsymbol{\Delta}_{i} \frac{\partial \boldsymbol{a}^{\mathrm{T}} \boldsymbol{D}_{i}^{\mathrm{T}} \boldsymbol{\Delta}_{i} \boldsymbol{A}_{i}^{-1 / 2} \boldsymbol{M}_{k} \boldsymbol{A}_{i}^{-1 / 2}}{\partial \boldsymbol{\mu}_{i}}
$$

For $I_{k}(\boldsymbol{\theta})$, one can write

$$
\begin{aligned}
I_{k}(\boldsymbol{\theta}) & =\frac{1}{n} \sum_{i=1}^{n} \boldsymbol{\zeta}_{i 1}^{\mathrm{T}}(\alpha) \boldsymbol{L}_{0, i}^{(k)} \boldsymbol{e}_{i}+\frac{1}{n} \sum_{i=1}^{n} \boldsymbol{\zeta}_{i 2}^{\mathrm{T}}(\boldsymbol{\gamma}) \boldsymbol{L}_{0, i}^{(k)} \boldsymbol{e}_{i}+\frac{1}{n} \sum_{i=1}^{n} \boldsymbol{\zeta}_{i 3}^{\mathrm{T}}(\boldsymbol{\beta}) \boldsymbol{L}_{0, i}^{(k)} \boldsymbol{e}_{i} \\
& =I_{k 1}(\alpha)+I_{k 2}(\boldsymbol{\gamma})+I_{k 3}(\boldsymbol{\beta}),
\end{aligned}
$$

where $\boldsymbol{L}_{0, i}^{(k)}$ is the value of $\boldsymbol{L}_{i}^{(k)}$ at $\boldsymbol{\mu}_{i}=\boldsymbol{\mu}_{0, i}$. Conditions (C5), (C6) and Lemma 6.2 of [1] indicate that $\sup _{1 \leq i \leq n, \boldsymbol{a}}\left\|\boldsymbol{L}_{0, i}^{(k)}\right\|=O_{P}\left(d_{n}^{1 / 2}\right)$. Furthermore, $I_{k}(\boldsymbol{\theta})$ has mean 0, and Condition $(\overline{\mathrm{C}} \overline{3})$ and Lemma S.2 ensure that

$$
\operatorname{Var}\left(I_{k 1}\right) \leq \frac{c d_{n}}{n} E\left\{\boldsymbol{\zeta}_{i 1}^{\mathrm{T}} \boldsymbol{e}_{i} \boldsymbol{e}_{i}^{\mathrm{T}} \boldsymbol{\zeta}_{i 1}\right\} \leq \frac{c d_{n}}{n} E\left(\boldsymbol{\zeta}_{i 1}^{\mathrm{T}} \boldsymbol{\zeta}_{i 1}\right)=O\left(n^{-1} J_{n}^{-2 r} d_{n} d_{x}\right)
$$

Therefore, $\sup _{\boldsymbol{a}, \boldsymbol{\theta} \in \Theta_{n}\left(C_{1}, C_{2}\right)}\left|I_{k 1}(\alpha)\right|=O_{P}\left(n^{-1 / 2} J_{n}^{-r} d_{n}^{1 / 2} d_{x}^{1 / 2}\right)$. Similarly, we can show that

$$
\sup _{\boldsymbol{a}, \boldsymbol{\theta} \in \Theta_{n}\left(C_{1}, C_{2}\right)}\left|I_{k 2}(\boldsymbol{\gamma})+I_{k 3}(\boldsymbol{\beta})\right|=O_{P}\left(n^{-1} d_{n}\right) .
$$

Let

$$
F_{i}^{*}=\left.\boldsymbol{\Delta}_{i}^{\mathrm{T}}\left\{\frac{\partial^{2} \boldsymbol{a}^{\mathrm{T}} \boldsymbol{D}_{i}^{\mathrm{T}} \boldsymbol{\Delta}_{i}^{\mathrm{T}}\left(\boldsymbol{\mu}_{i}\right) \boldsymbol{A}_{i}^{-1 / 2}\left(\boldsymbol{\mu}_{i}\right) \boldsymbol{M}_{k} \boldsymbol{A}_{i}^{-1 / 2}\left(\boldsymbol{\mu}_{i}\right)\left(\boldsymbol{y}_{i}-\boldsymbol{\mu}_{i}\right)}{\partial \boldsymbol{\mu}_{i} \partial \boldsymbol{\mu}_{i}^{\mathrm{T}}}\right\}\right|_{\mu_{i}=\mu_{i}^{*}} \boldsymbol{\Delta}_{i} .
$$

By [5], $\sup _{1 \leq i \leq n, \boldsymbol{a}}\left\|F_{i}^{*}\right\|=O_{P}\left(d_{n}^{1 / 2}\right)$. Then

$$
\begin{aligned}
R_{n}^{*}\left(\boldsymbol{\mu}^{*}\right)= & \frac{1}{2 n} \sum_{i=1}^{n} \boldsymbol{\zeta}_{i 1}^{\mathrm{T}}(\alpha) F_{i}^{*} \boldsymbol{\zeta}_{i 1}(\alpha)+\frac{1}{2 n} \sum_{i=1}^{n} \boldsymbol{\zeta}_{i 2}^{\mathrm{T}}(\boldsymbol{\gamma}) F_{i}^{*} \boldsymbol{\zeta}_{i 2}(\boldsymbol{\gamma}) \\
& +\frac{1}{2 n} \sum_{i=1}^{n} \boldsymbol{\zeta}_{i 3}^{\mathrm{T}}(\boldsymbol{\beta}) F_{i}^{*} \boldsymbol{\zeta}_{i 3}(\boldsymbol{\beta})+\frac{1}{n} \sum_{i=1}^{n} \boldsymbol{\zeta}_{i 1}^{\mathrm{T}}(\alpha) F_{i}^{*} \boldsymbol{\zeta}_{i 2}(\boldsymbol{\gamma}) \\
& +\frac{1}{n} \sum_{i=1}^{n} \boldsymbol{\zeta}_{i 1}^{\mathrm{T}}(\alpha) F_{i}^{*} \boldsymbol{\zeta}_{i 3}(\boldsymbol{\beta})+\frac{1}{n} \sum_{i=1}^{n} \boldsymbol{\zeta}_{i 2}^{\mathrm{T}}(\boldsymbol{\gamma}) F_{i}^{*} \boldsymbol{\zeta}_{i 3}(\boldsymbol{\beta}) \\
= & O_{P}\left(J_{n}^{-2 r} d_{n}^{1 / 2} d_{x}+n^{-1} d_{n}^{3 / 2}+n^{-1 / 2} J_{n}^{-r} d_{n} d_{x}^{1 / 2}\right)
\end{aligned}
$$


Thus,

$$
\begin{aligned}
\sup _{\boldsymbol{\theta} \in \Theta_{n}\left(C_{1}, C_{2}\right)} & \left\|\boldsymbol{G}_{n, k}(\boldsymbol{\theta})-\boldsymbol{G}_{n, k}^{0}(\boldsymbol{\theta})\right\| \\
= & \sup _{\boldsymbol{a}, \boldsymbol{\theta} \in \Theta_{n}\left(C_{1}, C_{2}\right)}\left|\boldsymbol{a}^{\mathrm{T}}\left\{\boldsymbol{G}_{n, k}(\boldsymbol{\theta})-\boldsymbol{G}_{n, k}^{0}(\boldsymbol{\theta})\right\}\right| \\
= & O_{P}\left(J_{n}^{-2 r} d_{n}^{1 / 2} d_{x}+n^{-1} d_{n}^{3 / 2}+n^{-1 / 2} J_{n}^{-r} d_{n} d_{x}^{1 / 2}\right) .
\end{aligned}
$$

Next, for any $\boldsymbol{a}=\left(\boldsymbol{a}_{0}^{\mathrm{T}}, \ldots, \boldsymbol{a}_{K}^{\mathrm{T}}\right)^{\mathrm{T}}$ with each $\boldsymbol{a}_{k} \in \boldsymbol{R}^{d_{n}}$ and $\boldsymbol{a}^{\mathrm{T}} \boldsymbol{a}=1$,

$$
\begin{gathered}
\boldsymbol{a}^{\mathrm{T}}\left\{\boldsymbol{C}_{n}(\boldsymbol{\theta})-\boldsymbol{C}_{n}^{0}(\boldsymbol{\theta})\right\} \boldsymbol{a}=\frac{1}{n} \sum_{i=1}^{n} \boldsymbol{a}^{\mathrm{T}}\left\{\boldsymbol{g}_{i}(\boldsymbol{\theta}) \boldsymbol{g}_{i}^{\mathrm{T}}(\boldsymbol{\theta})-\boldsymbol{g}_{i}^{0}(\boldsymbol{\theta}) \boldsymbol{g}_{i}^{0 \mathrm{~T}}(\boldsymbol{\theta})\right\} \boldsymbol{a} \\
=\sum_{k, k^{\prime}=1}^{K} \frac{1}{n} \sum_{i=1}^{n} \boldsymbol{a}_{k}^{\mathrm{T}}\left\{\boldsymbol{g}_{i, k}(\boldsymbol{\theta}) \boldsymbol{g}_{i, k^{\prime}}^{\mathrm{T}}(\boldsymbol{\theta})-\boldsymbol{g}_{i, k}^{0}(\boldsymbol{\theta}) \boldsymbol{g}_{i, k^{\prime}}^{0 \mathrm{~T}}(\boldsymbol{\theta})\right\} \boldsymbol{a}_{k^{\prime}}
\end{gathered}
$$

For any $k, k^{\prime}$,

$$
\begin{aligned}
& \frac{1}{n} \sum_{i=1}^{n} \boldsymbol{a}_{k}^{\mathrm{T}}\left\{\boldsymbol{g}_{i, k}(\boldsymbol{\theta}) \boldsymbol{g}_{i, k^{\prime}}^{\mathrm{T}}(\boldsymbol{\theta})-\boldsymbol{g}_{i, k}^{0}(\boldsymbol{\theta}) \boldsymbol{g}_{i, k^{\prime}}^{0 \mathrm{~T}}(\boldsymbol{\theta})\right\} \boldsymbol{a}_{k^{\prime}} \\
&=\frac{1}{n} \sum_{i=1}^{n}\left\{\boldsymbol{\zeta}_{i}^{\mathrm{T}}(\boldsymbol{\theta}) \boldsymbol{\Delta}_{0, i} \mathbf{L}_{0, i}^{(k)} \boldsymbol{e}_{i}+R_{n i}^{(k)}\left(\boldsymbol{\mu}^{*}\right)\right\}\left\{2 \boldsymbol{a}_{k^{\prime}}^{\mathrm{T}} \boldsymbol{D}_{i}^{\mathrm{T}} \boldsymbol{\Delta}_{0, i} \boldsymbol{V}_{0, i}^{\left(k^{\prime}\right)} \boldsymbol{e}_{i}\right. \\
&\left.\quad+2 \boldsymbol{a}_{k^{\prime}}^{\mathrm{T}} \boldsymbol{D}_{i}^{\mathrm{T}} \boldsymbol{\Gamma}_{0, i}^{(k)} \boldsymbol{\zeta}_{i}(\boldsymbol{\theta})+\boldsymbol{\zeta}_{i}^{\mathrm{T}}(\boldsymbol{\theta}) \boldsymbol{\Delta}_{0, i} \mathbf{L}_{0, i}^{\left(k^{\prime}\right)} \boldsymbol{e}_{i}+R_{n i}^{\left(k^{\prime}\right)}\left(\boldsymbol{\mu}^{* *}\right)\right\}^{\mathrm{T}} \\
&=O\left(n^{-1 / 2} d_{n}\right) .
\end{aligned}
$$

Finally, note that

$$
\begin{aligned}
& \frac{1}{n}\left|Q_{n}(\boldsymbol{\theta})-Q_{n}^{0}(\boldsymbol{\theta})\right| \\
&=\left|\boldsymbol{G}_{n}^{\mathrm{T}}(\boldsymbol{\theta}) \boldsymbol{C}_{n}^{-1}(\boldsymbol{\theta}) \boldsymbol{G}_{n}(\boldsymbol{\theta})-\left\{\boldsymbol{G}_{n}^{0}(\boldsymbol{\theta})\right\}^{\mathrm{T}}\left\{\boldsymbol{C}_{n}^{0}(\boldsymbol{\theta})\right\}^{-1} \boldsymbol{G}_{n}^{0}(\boldsymbol{\theta})\right| \\
& \leq\left|\left(\boldsymbol{G}_{n}-\boldsymbol{G}_{n}^{0}\right)^{\mathrm{T}}(\boldsymbol{\theta}) \boldsymbol{C}_{n}^{-1}(\boldsymbol{\theta})\left(\boldsymbol{G}_{n}-\boldsymbol{G}_{n}^{0}\right)(\boldsymbol{\theta})\right| \\
&+2\left|\left\{\boldsymbol{G}_{n}^{0}(\boldsymbol{\theta})\right\}^{\mathrm{T}}\left\{\boldsymbol{C}_{n}^{0}(\boldsymbol{\theta})\right\}^{-1}\left(\boldsymbol{G}_{n}-\boldsymbol{G}_{n}^{0}\right)(\boldsymbol{\theta})\right| \\
&+\left|\left\{\boldsymbol{G}_{n}^{0}(\boldsymbol{\theta})\right\}^{\mathrm{T}} \boldsymbol{C}_{n}^{-1}(\boldsymbol{\theta})\left[\boldsymbol{C}_{n}(\boldsymbol{\theta})-\boldsymbol{C}_{n}^{0}(\boldsymbol{\theta})\right]\left\{\boldsymbol{C}_{n}^{0}(\boldsymbol{\theta})\right\}^{-1} \boldsymbol{G}_{n}^{0}(\boldsymbol{\theta})\right| \\
& \leq \lambda_{\min }^{-1}\left\{\boldsymbol{C}_{n}(\boldsymbol{\theta})\right\}\left\|\left(\boldsymbol{G}_{n}-\boldsymbol{G}_{n}^{0}\right)(\boldsymbol{\theta})\right\|^{2} \\
&+2 \lambda_{\min }^{-1}\left\{\boldsymbol{C}_{n}^{0}(\boldsymbol{\theta})\right\}\left\|\boldsymbol{G}_{n}^{0}(\boldsymbol{\theta})\right\|\left\|\left(\boldsymbol{G}_{n}-\boldsymbol{G}_{n}^{0}\right)(\boldsymbol{\theta})\right\| \\
&+\lambda_{\min }^{-1}\left\{\boldsymbol{C}_{n}(\boldsymbol{\theta})\right\} \lambda_{\min }^{-1}\left\{\boldsymbol{C}_{n}^{0}(\boldsymbol{\theta})\right\} \lambda_{\max }\left\{\boldsymbol{C}_{n}(\boldsymbol{\theta})-\boldsymbol{C}_{n}^{0}(\boldsymbol{\theta})\right\}\left\|\boldsymbol{G}_{n}^{0}(\boldsymbol{\theta})\right\|^{2} .
\end{aligned}
$$

Thus, (S.10) follows. 
Next let

$$
\begin{aligned}
& \boldsymbol{J}_{\mathrm{DD}}^{(k)}=\frac{1}{n} \sum_{i=1}^{n} \boldsymbol{D}_{i}^{\mathrm{T}} \boldsymbol{\Gamma}_{0, i}^{(k)} \boldsymbol{D}_{i}, \quad \boldsymbol{J}_{\mathrm{DD}}=\left\{\left(\boldsymbol{J}_{\mathrm{DD}}^{(1)}\right)^{\mathrm{T}}, \ldots,\left(\boldsymbol{J}_{\mathrm{DD}}^{(K)}\right)^{\mathrm{T}}\right\}^{\mathrm{T}}, \\
& (\mathrm{S} .13) \boldsymbol{J}_{\mathrm{DZ}}^{(k)}=\frac{1}{n} \sum_{i=1}^{n} \boldsymbol{D}_{i}^{\mathrm{T}} \boldsymbol{\Gamma}_{0, i}^{(k)} \boldsymbol{Z}_{i}, \quad \boldsymbol{J}_{\mathrm{DZ}}=\left\{\left(\boldsymbol{J}_{\mathrm{DZ}}^{(1)}\right)^{\mathrm{T}}, \ldots,\left(\boldsymbol{J}_{\mathrm{DZ}}^{(K)}\right)^{\mathrm{T}}\right\}^{\mathrm{T}} \text {, and } \\
& (\mathrm{S} .14) \boldsymbol{J}_{\mathrm{DB}}^{(k)}=\frac{1}{n} \sum_{i=1}^{n} \boldsymbol{D}_{i}^{\mathrm{T}} \boldsymbol{\Gamma}_{0, i}^{(k)} \boldsymbol{B}_{i}, \quad \boldsymbol{J}_{\mathrm{DB}}=\left\{\left(\boldsymbol{J}_{\mathrm{DB}}^{(1)}\right)^{\mathrm{T}}, \ldots,\left(\boldsymbol{J}_{\mathrm{DB}}^{(K)}\right)^{\mathrm{T}}\right\}^{\mathrm{T}} \text {. }
\end{aligned}
$$

Lemma S.6. Under Conditions (C1)-(C8), for some $C_{1}$ and $C_{2}$ sufficiently large,

$$
\begin{gathered}
\sup _{\boldsymbol{\theta} \in \Theta\left(C_{1}, C_{2}\right)}\left\{\left\|\dot{\boldsymbol{G}}_{n}(\boldsymbol{\theta})-\boldsymbol{J}_{\mathrm{DD}}\right\|+\left\|\dot{\boldsymbol{G}}_{\boldsymbol{\beta}}(\boldsymbol{\theta})-\boldsymbol{J}_{\mathrm{DZ}}\right\|+\left\|\dot{\boldsymbol{G}}_{\boldsymbol{\gamma}}(\boldsymbol{\theta})-\boldsymbol{J}_{\mathrm{DB}}\right\|\right\} \\
=O_{P}\left(\sqrt{d_{n}^{3} / n}\right) .
\end{gathered}
$$

Proof. We only prove

$$
\sup _{\boldsymbol{\theta} \in \Theta\left(C_{1}, C_{2}\right)}\left\|\dot{\boldsymbol{G}}_{n}(\boldsymbol{\theta})-\boldsymbol{J}_{\mathrm{DD}}\right\|=O_{P}\left(\sqrt{d_{n}^{3} / n}\right),
$$

and the proofs of the other two terms are similar. Note that

$$
\begin{aligned}
& n \boldsymbol{a}^{\mathrm{T}}\left\{\dot{\boldsymbol{G}}_{n, k}(\boldsymbol{\theta})-\boldsymbol{J}_{\mathrm{DD}}^{(k)}\right\}=\sum_{i=1}^{n} \boldsymbol{a}^{\mathrm{T}} \boldsymbol{D}_{i}^{\mathrm{T}}\left(\boldsymbol{\Gamma}_{i}^{(k)}(\boldsymbol{\theta})-\boldsymbol{\Gamma}_{0, i}^{(k)}\right) \boldsymbol{D}_{i}+\sum_{i=1}^{n}\left[\boldsymbol{D}_{i}^{\mathrm{T}}\right. \\
& \left.\times \boldsymbol{\Delta}_{i}\left(\boldsymbol{\mu}_{i}\right)\left\{\frac{\partial}{\partial \boldsymbol{\mu}_{i}} \boldsymbol{a}^{\mathrm{T}} \boldsymbol{D}_{i}^{\mathrm{T}} \boldsymbol{\Delta}_{i}\left(\boldsymbol{\mu}_{i}\right) \boldsymbol{A}_{i}^{-1 / 2}\left(\boldsymbol{\mu}_{i}\right) \boldsymbol{M}_{k} \boldsymbol{A}_{i}^{-1 / 2}\left(\boldsymbol{\mu}_{i}\right)\right\}\left(\boldsymbol{Y}_{i}-\boldsymbol{\mu}_{i}\right)\right]^{\mathrm{T}} .
\end{aligned}
$$

Using the notation $\boldsymbol{L}_{i}^{(k)}$ defined in (S.11), we can write the transpose of the second term as

$$
\begin{aligned}
& \frac{1}{n} \sum_{i=1}^{n} \boldsymbol{D}_{i}^{\mathrm{T}} \boldsymbol{L}_{i}^{(k)}\left(\boldsymbol{\mu}_{i}\right)\left(\boldsymbol{Y}_{i}-\boldsymbol{\mu}_{i}\right)=\frac{1}{n} \sum_{i=1}^{n} \boldsymbol{D}_{i}^{\mathrm{T}} \boldsymbol{L}_{i}^{(k)}\left(\boldsymbol{\mu}_{i}\right) \\
& \times\left[\boldsymbol{e}_{i}+\boldsymbol{\Delta}_{0, i}\left\{\boldsymbol{\zeta}_{1 i}(\alpha)+\boldsymbol{\zeta}_{2 i}(\gamma)+\boldsymbol{\zeta}_{3 i}(\boldsymbol{\beta})\right\}\right] .
\end{aligned}
$$

Next, one has

$$
\sup _{\boldsymbol{a}, \boldsymbol{\theta} \in \Theta\left(C_{1}, C_{2}\right)}\left\|\frac{1}{n} \sum_{i=1}^{n} \boldsymbol{D}_{i}^{\mathrm{T}} \boldsymbol{L}_{i}^{(k)}\left(\boldsymbol{\mu}_{i}\right)\left(\boldsymbol{Y}_{i}-\boldsymbol{\mu}_{i}\right)\right\|=O_{P}\left(n^{-1 / 2} d_{n}^{3 / 2}+d_{x}^{1 / 2} d_{n} J_{n}^{-r}\right) .
$$


On the other hand,

$$
\begin{aligned}
& \sup _{\boldsymbol{a}, \boldsymbol{\theta} \in \Theta\left(C_{1}, C_{2}\right)}\left\|\frac{1}{n} \sum_{i=1}^{n} \boldsymbol{a}^{\mathrm{T}} \boldsymbol{D}_{i}^{\mathrm{T}}\left\{\boldsymbol{\Gamma}_{i}^{(k)}(\boldsymbol{\theta})-\boldsymbol{\Gamma}_{0, i}^{(k)}\right\} \boldsymbol{D}_{i}\right\| \\
& \quad=\sup _{\boldsymbol{a}, \boldsymbol{\theta} \in \Theta\left(C_{1}, C_{2}\right)}\left\|\frac{1}{n} \sum_{i=1}^{n}\left(\boldsymbol{\mu}_{i}-\boldsymbol{\mu}_{0, i}\right)^{\mathrm{T}} \frac{\partial}{\partial \boldsymbol{\mu}_{i}} \boldsymbol{a}^{\mathrm{T}} \boldsymbol{D}_{i}^{\mathrm{T}} \boldsymbol{\Gamma}_{i}^{(k)}\left(\boldsymbol{\mu}_{i}\right) \boldsymbol{D}_{i}\right\| \\
& =O_{P}\left(n^{-1 / 2} d_{n}+d_{x}^{1 / 2} d_{n}^{1 / 2} J_{n}^{-r}\right) .
\end{aligned}
$$

Hence (S.15) holds.

Lemma S.7. Under Conditions (C1)-(C8), one has

$$
\sup _{\boldsymbol{\theta} \in \Theta\left(C_{1}, C_{2}\right)}\left\|\boldsymbol{S}_{n}(\boldsymbol{\theta})\right\|=O_{P}\left(\rho_{n}^{-1} n^{-1 / 2} d_{n}^{1 / 2}\right) .
$$

Proof: According to Lemmas S.5, S.6 and the definition of $\boldsymbol{J}_{\mathrm{DD}}$ given in (S.12), one has

$$
\begin{aligned}
\boldsymbol{S}_{n}(\boldsymbol{\theta})= & \dot{\boldsymbol{G}}_{n}^{\mathrm{T}}(\boldsymbol{\theta}) \boldsymbol{C}_{n}^{-1}(\boldsymbol{\theta}) \boldsymbol{G}_{n}(\boldsymbol{\theta}) \\
= & \left\{\boldsymbol{J}_{\mathrm{DD}}+O_{P}\left(\sqrt{d_{n}^{3} / n}\right)\right\}^{\mathrm{T}} \boldsymbol{C}_{n}^{-1}(\boldsymbol{\theta})\left\{\boldsymbol{G}_{n}^{0}(\boldsymbol{\theta})+O_{P}\left(J_{n}^{-2 r} d_{n}^{1 / 2} d_{x}\right)\right. \\
& \left.+O_{P}\left(n^{-1} d_{n}^{3 / 2}\right)+O_{P}\left(n^{-1 / 2} J_{n}^{-r} d_{n} d_{x}^{1 / 2}\right)\right\} \\
= & \left\{\boldsymbol{J}_{\mathrm{DD}}+O_{P}\left(\sqrt{d_{n}^{3} / n}\right)\right\}^{\mathrm{T}}\left\{\boldsymbol{C}_{n}^{0}(\boldsymbol{\theta})\right\}^{-1}\left\{\boldsymbol{G}_{n}^{0}(\boldsymbol{\theta})++O_{P}\left(J_{n}^{-2 r} d_{n}^{1 / 2} d_{x}\right)\right. \\
& \left.+O_{P}\left(n^{-1} d_{n}^{3 / 2}\right)+O_{P}\left(n^{-1 / 2} J_{n}^{-r} d_{n} d_{x}^{1 / 2}\right)\right\} \\
& +\left\{\boldsymbol{J}_{\mathrm{DD}}+O_{P}\left(\sqrt{d_{n}^{3} / n}\right)\right\}^{\mathrm{T}} \boldsymbol{C}_{n}^{-1}(\boldsymbol{\theta})\left\{\boldsymbol{C}_{n}^{0}(\boldsymbol{\theta})-\boldsymbol{C}_{n}(\boldsymbol{\theta})\right\}\left\{\boldsymbol{C}_{n}^{0}(\boldsymbol{\theta})\right\}^{-1} \\
& \times\left\{\boldsymbol{G}_{n}^{0}(\boldsymbol{\theta})+O_{P}\left(J_{n}^{-2 r} d_{n}^{1 / 2} d_{x}+n^{-1} d_{n}^{3 / 2}+n^{-1 / 2} J_{n}^{-r} d_{n} d_{x}^{1 / 2}\right)\right\} .
\end{aligned}
$$

Lemma S.5 implies that the order of $\sup _{\boldsymbol{\theta} \in \Theta\left(C_{1}, C_{2}\right)}\left\|\boldsymbol{S}_{n}(\boldsymbol{\theta})\right\|$ is the same as that of $\sup _{\boldsymbol{\theta} \in \Theta\left(C_{1}, C_{2}\right)}\left\|\boldsymbol{J}_{\mathrm{DD}}\right\|\left\|\left\{\boldsymbol{C}_{n}^{0}(\boldsymbol{\theta})\right\}^{-1}\right\|\left\|\boldsymbol{G}_{n}^{0}(\boldsymbol{\theta})\right\|$. By Lemma S.4,

$$
\sup _{\boldsymbol{\theta} \in \Theta\left(C_{1}, C_{2}\right)}\left\|\boldsymbol{G}_{n}^{0}(\boldsymbol{\theta})\right\|=O_{P}\left(n^{-1 / 2} d_{n}^{1 / 2}\right), \quad \sup _{\boldsymbol{\theta} \in \Theta\left(C_{1}, C_{2}\right)} \lambda_{\min }\left\{\boldsymbol{C}_{n}^{0}(\boldsymbol{\theta})\right\} \geq c \rho_{n} .
$$

In addition, Lemma S.2 implies that $\boldsymbol{a}^{\mathrm{T}} \boldsymbol{J}_{\mathrm{DD}} \boldsymbol{a} \leq C$, except in an event whose probability tends to zero. Therefore, one has

$$
\sup _{\boldsymbol{\theta} \in \Theta\left(C_{1}, C_{2}\right)}\left\|\boldsymbol{J}_{\mathrm{DD}}\right\|\left\|\left\{\boldsymbol{C}_{n}^{0}(\boldsymbol{\theta})\right\}^{-1}\right\|\left\|\boldsymbol{G}_{n}^{0}(\boldsymbol{\theta})\right\|=O_{P}\left(\rho_{n}^{-1} n^{-1 / 2} d_{n}^{1 / 2}\right) .
$$

The desired result follows. 
Lemma S.8. Under Conditions (C1)-(C8), there exist constants $0<c \leq$ $C$, such that, for any vector $\boldsymbol{a}_{2}$ of length $d_{x} J_{n}$ and any $\boldsymbol{\theta} \in \Theta\left(C_{1}, C_{2}\right)$, as $n \rightarrow \infty, c \rho_{n}\left\|\boldsymbol{a}_{2}\right\|_{2}^{2} \leq \boldsymbol{a}_{2}^{\mathrm{T}} \boldsymbol{H}_{\boldsymbol{\gamma} \boldsymbol{\gamma}}(\boldsymbol{\theta}) \boldsymbol{a}_{2} \leq C \rho_{n}^{-1}\left\|\boldsymbol{a}_{2}\right\|_{2}^{2}$, except in an event whose probability tends to zero.

Proof. Note that

$$
\boldsymbol{a}_{2}^{\mathrm{T}} \boldsymbol{H}_{\boldsymbol{\gamma} \boldsymbol{\gamma}}(\boldsymbol{\theta}) \boldsymbol{a}_{2}=\boldsymbol{a}_{2}^{\mathrm{T}} \dot{\boldsymbol{G}}_{\boldsymbol{\gamma}}^{\mathrm{T}}(\boldsymbol{\theta}) \boldsymbol{C}_{n}^{-1}(\boldsymbol{\theta}) \dot{\boldsymbol{G}}_{\boldsymbol{\gamma}}(\boldsymbol{\theta}) \boldsymbol{a}_{2},
$$

and according to Lemma S.6, $\left\|\dot{\boldsymbol{G}}_{\boldsymbol{\gamma}}^{\mathrm{T}}(\boldsymbol{\theta})-\boldsymbol{J}_{\mathrm{DB}}\right\|=O_{P}\left(\sqrt{d_{n}^{3} / n}\right)$, where $\boldsymbol{J}_{\mathrm{DB}}$ is given in (S.14). Therefore,

$$
\begin{aligned}
\boldsymbol{a}_{2}^{\mathrm{T}} \boldsymbol{H}_{\boldsymbol{\gamma} \boldsymbol{\gamma}}(\boldsymbol{\theta}) \boldsymbol{a}_{2} & =\boldsymbol{a}_{2}^{\mathrm{T}}\left\{\boldsymbol{J}_{\mathrm{DB}}+O_{P}\left(\sqrt{d_{n}^{3} / n}\right)\right\}^{\mathrm{T}}\left\{\boldsymbol{C}_{n}^{0}(\boldsymbol{\theta})\right\}^{-1}\left\{\boldsymbol{J}_{\mathrm{DB}}+O_{P}\left(\sqrt{d_{n}^{3} / n}\right)\right\} \boldsymbol{a}_{2} \\
& +\boldsymbol{a}_{2}^{\mathrm{T}}\left\{\boldsymbol{J}_{\mathrm{DB}}+o P\left(\sqrt{d_{n}^{3} / n}\right)\right\}^{\mathrm{T}} \boldsymbol{C}_{n}^{-1}(\boldsymbol{\theta})\left(\boldsymbol{C}_{n}^{0}-\boldsymbol{C}_{n}\right)(\boldsymbol{\theta}) \\
& \times\left\{\boldsymbol{C}_{n}^{0}(\boldsymbol{\theta})\right\}^{-1}\left\{\boldsymbol{J}_{\mathrm{DB}}+O_{P}\left(\sqrt{d_{n}^{3} / n}\right)\right\} \boldsymbol{a}_{2} \\
& =\boldsymbol{a}_{2}^{\mathrm{T}} \boldsymbol{J}_{\mathrm{DB}}^{\mathrm{T}}\left\{\boldsymbol{C}_{n}^{0}(\boldsymbol{\theta})\right\}^{-1} \boldsymbol{J}_{\mathrm{DB}} \boldsymbol{a}_{2}\left\{1+o_{P}(1)\right\} .
\end{aligned}
$$

According to (S.5), one has

$$
c \boldsymbol{a}_{2}^{\mathrm{T}} \boldsymbol{J}_{\mathrm{DB}}^{\mathrm{T}} \boldsymbol{J}_{\mathrm{DB}} \boldsymbol{a}_{2} \leq \boldsymbol{a}_{2}^{\mathrm{T}} \boldsymbol{J}_{\mathrm{DB}}^{\mathrm{T}}\left\{\boldsymbol{C}_{n}^{0}(\boldsymbol{\theta})\right\}^{-1} \boldsymbol{J}_{\mathrm{DB}} \boldsymbol{a}_{2} \leq C \rho_{n}^{-1} \boldsymbol{a}_{2}^{\mathrm{T}} \boldsymbol{J}_{\mathrm{DB}}^{\mathrm{T}} \boldsymbol{J}_{\mathrm{DB}} \boldsymbol{a}_{2} .
$$

Thus the lemma follows from Lemma S.2.

Lemma S.9. Under Conditions (C1)-(C8), there exist constants $0<c \leq$ $C$, such that, for any $\boldsymbol{\theta} \in \Theta\left(C_{1}, C_{2}\right)$, as $n \rightarrow \infty, c \rho_{n} \leq \boldsymbol{a}^{\mathrm{T}} \boldsymbol{H}_{n}(\boldsymbol{\theta}) \boldsymbol{a} \leq C \rho_{n}^{-1}$, except in an event whose probability tends to zero.

Proof. We write $\boldsymbol{a}=\left(\boldsymbol{a}_{1}^{\mathrm{T}}, \boldsymbol{a}_{2}^{\mathrm{T}}\right)^{\mathrm{T}}$, where $\boldsymbol{a}_{1} \in R^{d_{z}}$ and $\boldsymbol{a}_{2} \in R^{d_{x} J_{n}}$. Note that $\boldsymbol{a}^{\mathrm{T}} \boldsymbol{H}_{n}(\boldsymbol{\theta}) \boldsymbol{a}=\boldsymbol{a}_{1}^{\mathrm{T}} \boldsymbol{H}_{\boldsymbol{\beta} \boldsymbol{\beta}}(\boldsymbol{\theta}) \boldsymbol{a}_{1}+\boldsymbol{a}_{2}^{\mathrm{T}} \boldsymbol{H}_{\boldsymbol{\gamma} \boldsymbol{\gamma}}(\boldsymbol{\theta}) \boldsymbol{a}_{2}+2 \boldsymbol{a}_{1}^{\mathrm{T}} \boldsymbol{H}_{\boldsymbol{\beta} \boldsymbol{\gamma}}(\boldsymbol{\theta}) \boldsymbol{a}_{2}$. According to Lemma S.8, $c \rho_{n}\left\|\boldsymbol{a}_{2}\right\|_{2}^{2} \leq \boldsymbol{a}_{2}^{\mathrm{T}} \boldsymbol{H}_{\boldsymbol{\gamma} \boldsymbol{\gamma}}(\boldsymbol{\theta}) \boldsymbol{a}_{2} \leq C \rho_{n}^{-1}\left\|\boldsymbol{a}_{2}\right\|_{2}^{2}$, a.s. Similar to the proof of Lemma S.8, we could have $c\left\|\boldsymbol{a}_{1}\right\|_{2}^{2} \leq \boldsymbol{a}_{1}^{\mathrm{T}} \boldsymbol{H}_{\boldsymbol{\beta} \boldsymbol{\beta}}(\boldsymbol{\theta}) \boldsymbol{a}_{1} \leq C\left\|\boldsymbol{a}_{1}\right\|_{2}^{2}$, a.s. Note that

$$
\boldsymbol{a}_{1}^{\mathrm{T}} \boldsymbol{H}_{\boldsymbol{\beta} \boldsymbol{\gamma}}(\boldsymbol{\theta}) \boldsymbol{a}_{2}=\boldsymbol{a}_{1}^{\mathrm{T}} \boldsymbol{J}_{\mathrm{DZ}}^{\mathrm{T}}\left(\boldsymbol{C}_{n}^{0}\right)^{-1} \boldsymbol{J}_{\mathrm{DB}} \boldsymbol{a}_{2}\left\{1+o_{P}(1)\right\},
$$

and

$$
\boldsymbol{a}_{1}^{\mathrm{T}} \boldsymbol{J}_{\mathrm{DZ}}^{\mathrm{T}} \boldsymbol{J}_{\mathrm{DB}} \boldsymbol{a}_{2}=\sum_{k=1}^{K} \boldsymbol{a}_{1}^{\mathrm{T}}\left(\boldsymbol{J}_{\mathrm{DZ}}^{(k)}\right)^{\mathrm{T}} \boldsymbol{J}_{\mathrm{DB}}^{(k)} \boldsymbol{a}_{2}=\sum_{k=1}^{K} \frac{1}{n^{2}} \sum_{i, i^{\prime}=1}^{n} \boldsymbol{a}_{1}^{\mathrm{T}} \boldsymbol{Z}_{i}^{\mathrm{T}} \boldsymbol{\Gamma}_{0, i}^{(k)} \boldsymbol{D}_{i} \boldsymbol{D}_{i^{\prime}}^{\mathrm{T}} \boldsymbol{\Gamma}_{0, i^{\prime}}^{(k)} \boldsymbol{B}_{i^{\prime}} \boldsymbol{a}_{2},
$$




$$
\begin{aligned}
\boldsymbol{a}_{1}^{\mathrm{T}} \boldsymbol{Z}_{i}^{\mathrm{T}} \boldsymbol{\Gamma}_{0, i}^{(k)} \boldsymbol{D}_{i} \boldsymbol{D}_{i^{\prime}}^{\mathrm{T}} \boldsymbol{\Gamma}_{0, i^{\prime}}^{(k)} \boldsymbol{B}_{i^{\prime}} \boldsymbol{a}_{2}=\boldsymbol{a}_{1}^{\mathrm{T}} \boldsymbol{Z}_{i}^{\mathrm{T}} \boldsymbol{\Gamma}_{0, i}^{(k)} \boldsymbol{Z}_{i} \boldsymbol{Z}_{i^{\prime}}^{\mathrm{T}} \boldsymbol{\Gamma}_{0, i^{\prime}}^{(k)} \boldsymbol{B}_{i^{\prime}} \boldsymbol{a}_{2} \\
+\boldsymbol{a}_{1}^{\mathrm{T}} \boldsymbol{Z}_{i}^{\mathrm{T}} \boldsymbol{\Gamma}_{0, i}^{(k)} \boldsymbol{B}_{i} \boldsymbol{B}_{i^{\prime}}^{\mathrm{T}} \boldsymbol{\Gamma}_{0, i^{\prime}}^{(k)} \boldsymbol{B}_{i^{\prime}} \boldsymbol{a}_{2},
\end{aligned}
$$

thus $\left|\boldsymbol{a}_{1}^{\mathrm{T}} \boldsymbol{H}_{\boldsymbol{\beta} \boldsymbol{\gamma}}(\boldsymbol{\theta}) \boldsymbol{a}_{2}\right|=o\left(\left\|\boldsymbol{a}_{1}\right\|\left\|\boldsymbol{a}_{2}\right\|\right)$, a.s. Hence,

$$
c \rho_{n} \leq \boldsymbol{a}^{\mathrm{T}} \boldsymbol{H}_{n}(\boldsymbol{\theta}) \boldsymbol{a} \leq C \rho_{n}^{-1}, \quad \text { a.s. }
$$

S.2. Asymptotic results with best approximation. The following lemma shows the consistency of $\widehat{\boldsymbol{\theta}}^{\mathrm{QIF}}$.

Lemma S.10. Under Conditions (C1)-(C8),

$$
\left\|\widehat{\boldsymbol{\theta}}^{\mathrm{QIF}}-\widetilde{\boldsymbol{\theta}}_{0}\right\|=O_{P}\left(n^{-1 / 2} d_{n}^{1 / 2}\right)
$$

Proof. We first show that for any $\varepsilon>0$, there exists a $C=C(\varepsilon)$, such that

$$
P\left\{\inf _{\left\|\boldsymbol{\theta}-\widetilde{\boldsymbol{\theta}}_{0}\right\|=C n^{-1 / 2} d_{n}^{1 / 2}} \boldsymbol{Q}_{n}^{0}(\boldsymbol{\theta})>\boldsymbol{Q}_{n}^{0}\left(\widetilde{\boldsymbol{\theta}}_{0}\right)\right\}>1-\varepsilon,
$$

as $n \rightarrow \infty$. Let $\boldsymbol{U}_{n}(\boldsymbol{\theta})=\boldsymbol{G}_{n}^{0}(\boldsymbol{\theta})-\boldsymbol{G}_{n}^{0}\left(\widetilde{\boldsymbol{\theta}}_{0}\right)$. Then

$$
\begin{aligned}
n^{-1}\left\{\boldsymbol{Q}_{n}^{0}(\boldsymbol{\theta})\right. & \left.-\boldsymbol{Q}_{n}^{0}\left(\widetilde{\boldsymbol{\theta}}_{0}\right)\right\}=\boldsymbol{U}_{n}^{\mathrm{T}}(\boldsymbol{\theta})\left(\boldsymbol{C}_{n}^{0}\right)^{-1} \boldsymbol{U}_{n}(\boldsymbol{\theta})+2 \boldsymbol{U}_{n}^{\mathrm{T}}(\boldsymbol{\theta})\left(\boldsymbol{C}_{n}^{0}\right)^{-1} \boldsymbol{G}_{n}^{0}\left(\widetilde{\boldsymbol{\theta}}_{0}\right) \\
& +\left\{\boldsymbol{G}_{n}^{0}(\boldsymbol{\theta})\right\}^{\mathrm{T}}\left(\boldsymbol{C}_{n}^{0}(\boldsymbol{\theta})\right)^{-1}\left\{\boldsymbol{C}_{n}^{0}-\boldsymbol{C}_{n}^{0}(\boldsymbol{\theta})\right\}\left(\boldsymbol{C}_{n}^{0}\right)^{-1} \boldsymbol{G}_{n}^{0}(\boldsymbol{\theta}) .
\end{aligned}
$$

By Lemma S.3, there exists a constant $c_{11}>0$ such that for any $\boldsymbol{\theta} \in \Theta_{n}(C)$,

$$
\begin{gathered}
\left|\boldsymbol{U}_{n}^{\mathrm{T}}(\boldsymbol{\theta})\left(\boldsymbol{C}_{n}^{0}\right)^{-1} \boldsymbol{G}_{n}^{0}\left(\widetilde{\boldsymbol{\theta}}_{0}\right)\right| \leq c_{11} \rho_{n}^{-1} \sum_{k=1}^{K}\left|\left\{g_{k 3}^{0}(\boldsymbol{\gamma})+g_{k 4}^{0}(\boldsymbol{\beta})\right\}^{\mathrm{T}}\left\{g_{k 1}^{0}+g_{k 2}^{0}(\alpha)\right\}\right| \\
\leq c_{11} \rho_{n}^{-1} \sum_{k=1}^{K}\left(\left\|g_{k 3}^{0}(\boldsymbol{\gamma})\right\|+\left\|g_{k 4}^{0}(\boldsymbol{\beta})\right\|\right)\left(\left\|g_{k 1}^{0}\right\|+\left\|g_{k 2}^{0}(\alpha)\right\|\right) .
\end{gathered}
$$

Furthermore, Lemma S.3 entails that there exists a constant $C_{1}>0$ such that

$$
\left|\boldsymbol{U}_{n}^{\mathrm{T}}(\boldsymbol{\theta})\left(\boldsymbol{C}_{n}^{0}\right)^{-1} \boldsymbol{G}_{n}^{0}\left(\widetilde{\boldsymbol{\theta}}_{0}\right)\right| \leq C C_{1}\left(d_{x}^{1 / 2} h^{r}+1 / \sqrt{n}\right) \rho_{n}^{-1} n^{-1 / 2} d_{n}^{1 / 2}
$$

when $n$ is large enough. On the other hand, Condition $(\mathrm{C} 7)$ entails that there exists a $c_{12}>0$, such that

$$
\boldsymbol{U}_{n}^{\mathrm{T}}(\boldsymbol{\theta})\left(\boldsymbol{C}_{n}^{0}\right)^{-1} \boldsymbol{U}_{n}(\boldsymbol{\theta}) \geq c_{12} \sum_{k=1}^{K}\left\|g_{k 3}^{0}(\boldsymbol{\gamma})+g_{k 4}^{0}(\boldsymbol{\beta})\right\|^{2},
$$


and Lemma S.3 indicates that there exists a constant $C_{2}>0$ such that

$$
\boldsymbol{U}_{n}^{\mathrm{T}}(\boldsymbol{\theta})\left(\boldsymbol{C}_{n}^{0}\right)^{-1} \boldsymbol{U}_{n}(\boldsymbol{\theta}) \geq C^{2} C_{2} n^{-1} d_{n} .
$$

Therefore we prove (S.16) by choosing $C$ sufficiently large such that $C>$ $C_{1} / C_{2}$. Equations (S.10) and (S.16) together imply that

$$
P\left\{\inf _{\|\boldsymbol{\theta}-\widetilde{\boldsymbol{\theta}}\|=C n^{-1 / 2} d_{n}^{1 / 2}} \boldsymbol{Q}_{n}(\boldsymbol{\theta})>\boldsymbol{Q}_{n}\left(\widetilde{\boldsymbol{\theta}}_{0}\right)\right\}>1-\varepsilon .
$$

Next

$$
\begin{aligned}
& P\left\{\left\|\widehat{\boldsymbol{\theta}}^{\mathrm{QIF}}-\widetilde{\boldsymbol{\theta}}_{0}\right\| \leq C n^{-1 / 2} d_{n}^{1 / 2}\right\} \geq P\left\{\inf _{\left\|\boldsymbol{\theta}-\widetilde{\boldsymbol{\theta}}_{0}\right\|=C n^{-1 / 2} d_{n}^{1 / 2}} \boldsymbol{Q}_{n}(\boldsymbol{\theta})>\boldsymbol{Q}_{n}\left(\widetilde{\boldsymbol{\theta}}_{0}\right)\right\} \\
& >1-\varepsilon
\end{aligned}
$$

which entails that $\left\|\widehat{\boldsymbol{\theta}}^{\mathrm{QIF}}-\widetilde{\boldsymbol{\theta}}_{0}\right\|=O_{P}\left(n^{-1 / 2} d_{n}^{1 / 2}\right)$.

Lemma S.11. Under Conditions (C1)-(C8),

$$
\boldsymbol{H}_{\boldsymbol{\beta} \boldsymbol{\beta}}\left(\widetilde{\boldsymbol{\theta}}_{0}\right)-\boldsymbol{H}_{\boldsymbol{\beta} \boldsymbol{\gamma}}\left(\widetilde{\boldsymbol{\theta}}_{0}\right)\left\{\boldsymbol{H}_{\boldsymbol{\gamma} \boldsymbol{\gamma}}\left(\widetilde{\boldsymbol{\theta}}_{0}\right)\right\}^{-1} \boldsymbol{H}_{\boldsymbol{\gamma} \boldsymbol{\beta}}\left(\widetilde{\boldsymbol{\theta}}_{0}\right)=\widehat{\boldsymbol{J}}_{\mathrm{DZ}}^{\mathrm{T}}\left(\boldsymbol{C}_{n}^{0}\right)^{-1} \widehat{\boldsymbol{J}}_{\mathrm{DZ}}\left\{1+o_{P}(1)\right\},
$$

where $\widetilde{\boldsymbol{\theta}}_{0}=\left(\boldsymbol{\beta}_{0}^{\mathrm{T}}, \widetilde{\boldsymbol{\gamma}}^{\mathrm{T}}\right)^{\mathrm{T}}$ and $\widehat{\boldsymbol{J}}_{\mathrm{Dz}}$ is given in (2.7).

Proof. According to the notation in (A.7), one has

$$
\begin{aligned}
& \boldsymbol{H}_{\boldsymbol{\beta} \boldsymbol{\beta}}(\boldsymbol{\theta})-\boldsymbol{H}_{\boldsymbol{\beta} \boldsymbol{\gamma}}(\boldsymbol{\theta})\left\{\boldsymbol{H}_{\boldsymbol{\gamma} \boldsymbol{\gamma}}(\boldsymbol{\theta})\right\}^{-1} \boldsymbol{H}_{\boldsymbol{\gamma} \boldsymbol{\beta}}(\boldsymbol{\theta})=\dot{\boldsymbol{G}}_{\boldsymbol{\beta}}^{\mathrm{T}}(\boldsymbol{\theta}) \boldsymbol{C}_{n}^{-1}(\boldsymbol{\theta}) \dot{\boldsymbol{G}}_{\boldsymbol{\beta}}(\boldsymbol{\theta}) \\
& -\dot{\boldsymbol{G}}_{\boldsymbol{\beta}}^{\mathrm{T}}(\boldsymbol{\theta}) \boldsymbol{C}_{n}^{-1}(\boldsymbol{\theta}) \dot{\boldsymbol{G}}_{\boldsymbol{\gamma}}(\boldsymbol{\theta})\left\{\dot{\boldsymbol{G}}_{\boldsymbol{\gamma}}^{\mathrm{T}}(\boldsymbol{\theta}) \boldsymbol{C}_{n}^{-1}(\boldsymbol{\theta}) \dot{\boldsymbol{G}}_{\boldsymbol{\gamma}}(\boldsymbol{\theta})\right\} \dot{\boldsymbol{G}}_{\boldsymbol{\gamma}}^{\mathrm{T}}(\boldsymbol{\theta}) \boldsymbol{C}_{n}^{-1}(\boldsymbol{\theta}) \dot{\boldsymbol{G}}_{\boldsymbol{\beta}}(\boldsymbol{\theta}) .
\end{aligned}
$$

Lemma S.6 implies that

$$
\boldsymbol{H}_{\boldsymbol{\beta} \boldsymbol{\beta}}\left(\widetilde{\boldsymbol{\theta}}_{0}\right)=\dot{\boldsymbol{G}}_{\boldsymbol{\beta}}^{\mathrm{T}}\left(\widetilde{\boldsymbol{\theta}}_{0}\right)\left(\boldsymbol{C}_{n}^{0}\right)^{-1} \dot{\boldsymbol{G}}_{\boldsymbol{\beta}}\left(\widetilde{\boldsymbol{\theta}}_{0}\right)\left\{1+o_{P}(1)\right\}=\boldsymbol{J}_{\mathrm{DZ}}^{\mathrm{T}}\left(\boldsymbol{C}_{n}^{0}\right)^{-1} \boldsymbol{J}_{\mathrm{DZ}}\left\{1+o_{P}(1)\right\},
$$

where $\boldsymbol{J}_{\mathrm{DZ}}$ in $(\mathrm{S} .13)$, i.e.,

$$
\boldsymbol{H}_{\boldsymbol{\beta} \boldsymbol{\beta}}\left(\widetilde{\boldsymbol{\theta}}_{0}\right)=\frac{1}{n^{2}} \sum_{i=1}^{n}\left(\begin{array}{c}
\boldsymbol{D}_{i}^{\mathrm{T}} \boldsymbol{\Gamma}_{0, i}^{(1)} \boldsymbol{Z}_{i} \\
\vdots \\
\boldsymbol{D}_{i}^{\mathrm{T}} \boldsymbol{\Gamma}_{0, i}^{(K)} \boldsymbol{Z}_{i}
\end{array}\right)^{\mathrm{T}}\left(\boldsymbol{C}_{n}^{0}\right)^{-1} \sum_{i=1}^{n}\left(\begin{array}{c}
\boldsymbol{D}_{i}^{\mathrm{T}} \boldsymbol{\Gamma}_{0, i}^{(1)} \boldsymbol{Z}_{i} \\
\vdots \\
\boldsymbol{D}_{i}^{\mathrm{T}} \boldsymbol{\Gamma}_{0, i}^{(K)} \boldsymbol{Z}_{i}
\end{array}\right)\left\{1+o_{P}(1)\right\}
$$

Similarly, Lemma S.6 implies that

$$
\boldsymbol{H}_{\boldsymbol{\beta} \gamma}\left(\widetilde{\boldsymbol{\theta}}_{0}\right)=\dot{\boldsymbol{G}}_{\boldsymbol{\beta}}^{\mathrm{T}}\left(\widetilde{\boldsymbol{\theta}}_{0}\right)\left(\boldsymbol{C}_{n}^{0}\right)^{-1} \dot{\boldsymbol{G}}_{\boldsymbol{\gamma}}\left(\widetilde{\boldsymbol{\theta}}_{0}\right)\left\{1+o_{P}(1)\right\}=\boldsymbol{J}_{\mathrm{DZ}}^{\mathrm{T}}\left(\boldsymbol{C}_{n}^{0}\right)^{-1} \boldsymbol{J}_{\mathrm{DB}}\left\{1+o_{P}(1)\right\},
$$


where $\boldsymbol{J}_{\mathrm{DB}}$ in (S.14), i.e.,

$$
\boldsymbol{H}_{\boldsymbol{\beta} \boldsymbol{\gamma}}\left(\widetilde{\boldsymbol{\theta}}_{0}\right)=\frac{1}{n^{2}} \sum_{i=1}^{n}\left(\begin{array}{c}
\boldsymbol{D}_{i}^{\mathrm{T}} \boldsymbol{\Gamma}_{0, i}^{(1)} \boldsymbol{Z}_{i} \\
\vdots \\
\boldsymbol{D}_{i}^{\mathrm{T}} \boldsymbol{\Gamma}_{0, i}^{(K)} \boldsymbol{Z}_{i}
\end{array}\right)^{\mathrm{T}}\left(\boldsymbol{C}_{n}^{0}\right)^{-1} \sum_{i=1}^{n}\left(\begin{array}{c}
\boldsymbol{D}_{i}^{\mathrm{T}} \boldsymbol{\Gamma}_{0, i}^{(1)} \boldsymbol{B}_{i} \\
\vdots \\
\boldsymbol{D}_{i}^{\mathrm{T}} \boldsymbol{\Gamma}_{0, i}^{(K)} \boldsymbol{B}_{i}
\end{array}\right)\left\{1+o_{P}(1)\right\} .
$$

Therefore, letting

$$
\widehat{\boldsymbol{Z}}_{i}=\boldsymbol{Z}_{i}-\boldsymbol{B}_{i}\left\{\boldsymbol{J}_{\boldsymbol{D} \boldsymbol{B}}^{\mathrm{T}}\left(\boldsymbol{C}_{n}^{0}\right)^{-1} \boldsymbol{J}_{\mathrm{DB}}\right\}^{-1}\left\{\boldsymbol{J}_{\mathrm{DZ}}^{\mathrm{T}}\left(\boldsymbol{C}_{n}^{0}\right)^{-1} \boldsymbol{J}_{\mathrm{DB}}\right\}^{\mathrm{T}},
$$

we have

$$
\begin{aligned}
& \boldsymbol{H}_{\boldsymbol{\beta} \boldsymbol{\beta}}\left(\widetilde{\boldsymbol{\theta}}_{0}\right)-\boldsymbol{H}_{\boldsymbol{\beta} \boldsymbol{\gamma}}\left(\widetilde{\boldsymbol{\theta}}_{0}\right)\left\{\boldsymbol{H}_{\boldsymbol{\gamma} \boldsymbol{\gamma}}\left(\widetilde{\boldsymbol{\theta}}_{0}\right)\right\}^{-1} \boldsymbol{H}_{\boldsymbol{\gamma} \boldsymbol{\beta}}\left(\widetilde{\boldsymbol{\theta}}_{0}\right) \\
& =\frac{1}{n^{2}} \sum_{i=1}^{n}\left(\begin{array}{c}
\boldsymbol{D}_{i}^{\mathrm{T}} \boldsymbol{\Gamma}_{0, i}^{(1)} \widehat{\boldsymbol{Z}}_{i} \\
\vdots \\
\boldsymbol{D}_{i}^{\mathrm{T}} \boldsymbol{\Gamma}_{0, i}^{(K)} \widehat{\boldsymbol{Z}}_{i}
\end{array}\right)\left(\boldsymbol{C}_{n}^{0}\right)^{-1} \sum_{i=1}^{n}\left(\begin{array}{c}
\boldsymbol{D}_{i}^{\mathrm{T}} \boldsymbol{\Gamma}_{0, i}^{(1)} \widehat{\boldsymbol{Z}}_{i} \\
\vdots \\
\boldsymbol{D}_{i}^{\mathrm{T}} \boldsymbol{\Gamma}_{0, i}^{(K)} \widehat{\boldsymbol{Z}}_{i}
\end{array}\right)\left\{1+o_{P}(1)\right\} .
\end{aligned}
$$

The result follows from (S.13).

In the following, let $\mathbb{X}$ and $\mathbb{Z}$ be the collection of all $\boldsymbol{X}_{i t}$ 's and $\boldsymbol{Z}_{i t}$ 's, respectively, i.e.,

$$
\mathbb{X}=\left(\boldsymbol{X}_{1}^{\mathrm{T}}, \ldots, \boldsymbol{X}_{n}^{\mathrm{T}}\right), \quad \mathbb{Z}=\left(\boldsymbol{Z}_{1}^{\mathrm{T}}, \ldots, \boldsymbol{Z}_{n}^{\mathrm{T}}\right)
$$

Lemma S.12. Under Conditions (C1)-(C8),

$$
n^{1 / 2} \boldsymbol{A}_{n} \boldsymbol{\Sigma}_{n}^{-1 / 2} \boldsymbol{\Psi}_{n}^{-1}\left(\dot{\boldsymbol{G}}_{\boldsymbol{\beta}}^{\mathrm{T}}\left(\widetilde{\boldsymbol{\theta}}_{0}\right)-\boldsymbol{H}_{\boldsymbol{\beta} \boldsymbol{\gamma}}\left(\widetilde{\boldsymbol{\theta}}_{0}\right) \boldsymbol{H}_{\boldsymbol{\gamma} \gamma}^{-1}\left(\widetilde{\boldsymbol{\theta}}_{0}\right) \dot{\boldsymbol{G}}_{\boldsymbol{\gamma}}^{\mathrm{T}}\left(\widetilde{\boldsymbol{\theta}}_{0}\right)\right) \boldsymbol{C}_{n}^{-1}\left(\widetilde{\boldsymbol{\theta}}_{0}\right) \boldsymbol{G}_{n}\left(\widetilde{\boldsymbol{\theta}}_{0}\right)
$$

$\stackrel{D}{\rightarrow} N\left(0, \boldsymbol{\Sigma}_{A}\right)$, where $\boldsymbol{A}_{n}$ is any $q \times d_{z}$ matrix with a finite $q$ such that $\boldsymbol{\Sigma}_{A}=$ $\lim _{n \rightarrow \infty} \boldsymbol{A}_{n}^{\otimes 2}$, and $\boldsymbol{\Sigma}_{n}=\widehat{\boldsymbol{\Psi}}_{n}^{-1} \widehat{\boldsymbol{\Omega}}_{n} \widehat{\boldsymbol{\Psi}}_{n}^{-1}$ with $\boldsymbol{\Omega}_{n}$ and $\widehat{\boldsymbol{\Psi}}_{n}$ given in (2.9).

Proof. According to Lemma S.6, we have $\left\|\dot{\boldsymbol{G}}_{\boldsymbol{\beta}}^{\mathrm{T}}\left(\widetilde{\boldsymbol{\theta}}_{0}\right)-\boldsymbol{J}_{\mathrm{DZ}}\right\|=O_{P}\left(\sqrt{d_{n}^{3} / n}\right)$ and $\left\|\dot{\boldsymbol{G}}_{\boldsymbol{\gamma}}^{\mathrm{T}}\left(\widetilde{\boldsymbol{\theta}}_{0}\right)-\boldsymbol{J}_{\mathrm{DB}}\right\|=O_{P}\left(\sqrt{d_{n}^{3} / n}\right)$. Hence,

$$
\dot{\boldsymbol{G}}_{\boldsymbol{\beta}}^{\mathrm{T}}\left(\widetilde{\boldsymbol{\theta}}_{0}\right)-\boldsymbol{H}_{\boldsymbol{\beta} \boldsymbol{\gamma}}\left(\widetilde{\boldsymbol{\theta}}_{0}\right) \boldsymbol{H}_{\boldsymbol{\gamma} \boldsymbol{\gamma}}^{-1}\left(\widetilde{\boldsymbol{\theta}}_{0}\right) \dot{\boldsymbol{G}}_{\boldsymbol{\gamma}}^{\mathrm{T}}\left(\widetilde{\boldsymbol{\theta}}_{0}\right)=\widehat{\boldsymbol{J}}_{\mathrm{DZ}}+O_{P}\left(\sqrt{d_{n}^{3} / n}\right),
$$

where $\widehat{\boldsymbol{J}}_{\mathrm{DZ}}$ is given in (2.7). Using similar arguments as in the proof of Lemma 
S.5, we can show that $\left\|\boldsymbol{G}_{n}\left(\widetilde{\boldsymbol{\theta}}_{0}\right)-\boldsymbol{G}_{n}^{0}\left(\widetilde{\boldsymbol{\theta}}_{0}\right)\right\|=o_{P}\left(h^{r}\right)$. Thus,

$$
\begin{aligned}
& \sqrt{n} \boldsymbol{A}_{n} \boldsymbol{\Sigma}_{n}^{-1 / 2} \boldsymbol{\Psi}_{n}^{-1}\left\{\dot{\boldsymbol{G}}_{\boldsymbol{\beta}}^{\mathrm{T}}\left(\widetilde{\boldsymbol{\theta}}_{0}\right)-\boldsymbol{H}_{\boldsymbol{\beta} \boldsymbol{\gamma}}\left(\widetilde{\boldsymbol{\theta}}_{0}\right) \boldsymbol{H}_{\boldsymbol{\gamma} \gamma}^{-1}\left(\widetilde{\boldsymbol{\theta}}_{0}\right) \dot{\boldsymbol{G}}_{\boldsymbol{\gamma}}^{\mathrm{T}}\left(\widetilde{\boldsymbol{\theta}}_{0}\right)\right\}^{\mathrm{T}} \boldsymbol{C}_{n}^{-1}\left(\widetilde{\boldsymbol{\theta}}_{0}\right) \boldsymbol{G}_{n}\left(\widetilde{\boldsymbol{\theta}}_{0}\right) \\
&=\sqrt{n} \boldsymbol{A}_{n} \boldsymbol{\Sigma}_{n}^{-1 / 2} \boldsymbol{\Psi}_{n}^{-1}\left\{\widehat{\boldsymbol{J}}_{\mathrm{DZ}}+O_{P}\left(\sqrt{d_{n}^{3} / n}\right)\right\}^{\mathrm{T}}\left(\boldsymbol{C}_{n}^{0}\right)^{-1}\left\{\boldsymbol{G}_{n}^{0}+O_{P}\left(d_{x} J_{n}^{-r}\right)\right\} \\
&+\sqrt{n} \boldsymbol{A}_{n} \boldsymbol{\Sigma}_{n}^{-1 / 2} \boldsymbol{\Psi}_{n}^{-1}\left\{\widehat{\boldsymbol{J}}_{\mathrm{DZ}}+O_{P}\left(\sqrt{d_{n}^{3} / n}\right)\right\}^{\mathrm{T}}\left(\boldsymbol{C}_{n}^{0}\left(\widetilde{\boldsymbol{\theta}}_{0}\right)\right)^{-1} \\
& \times\left\{\boldsymbol{C}_{n}^{0}-\boldsymbol{C}_{n}^{0}\left(\widetilde{\boldsymbol{\theta}}_{0}\right)\right\}\left(\boldsymbol{C}_{n}^{0}\right)^{-1}\left\{\boldsymbol{G}_{n}^{0}+O_{P}\left(d_{x}^{1 / 2} J_{n}^{-r}\right)\right\} \\
&=\sqrt{n} \boldsymbol{A}_{n} \boldsymbol{\Sigma}_{n}^{-1 / 2} \boldsymbol{\Psi}_{n}^{-1}\left\{\widehat{\boldsymbol{J}}_{\mathrm{DZ}}^{\mathrm{T}}\left(\boldsymbol{C}_{n}^{0}\right)^{-1} \boldsymbol{G}_{n}^{0}+O_{P}\left(n^{-1} d_{n}^{2} \rho_{n}^{-1}\right)\right. \\
&\left.\quad+O_{P}\left(n^{-1 / 2} d_{n}^{3 / 2} \rho_{n}^{-1} d_{x} J_{n}^{-r}\right)\right\} \\
&=\sqrt{n} \boldsymbol{A}_{n} \boldsymbol{\Sigma}_{n}^{-1 / 2} \boldsymbol{\Psi}_{n}^{-1}\left\{\widehat{\boldsymbol{J}}_{\mathrm{DZ}}^{\mathrm{T}}\left(\boldsymbol{C}_{n}^{0}\right)^{-1} \boldsymbol{G}_{n}^{0}+o_{P}\left(n^{-1 / 2}\right)\right\} .
\end{aligned}
$$

Next we write $\left(\boldsymbol{C}_{n}^{0}\right)^{-1}=\left(\boldsymbol{C}_{k k^{\prime}}^{0}\right)_{1 \leq k, k^{\prime} \leq K}$, where $\boldsymbol{C}_{k k^{\prime}}^{0}$ is a submatrix of dimension $d_{n} \times d_{n}$, for any $1 \leq k, k^{\prime} \leq \bar{K}$. Thus,

$$
\begin{aligned}
\widehat{\boldsymbol{J}}_{\mathrm{DZ}}^{\mathrm{T}}\left(\boldsymbol{C}_{n}^{0}\right)^{-1} \boldsymbol{G}_{n}^{0} & =\frac{1}{n^{2}} \sum_{i=1}^{n}\left(\begin{array}{c}
\boldsymbol{D}_{i}^{\mathrm{T}} \boldsymbol{\Gamma}_{0, i}^{(1)} \widehat{\boldsymbol{Z}}_{i} \\
\vdots \\
\boldsymbol{D}_{i}^{\mathrm{T}} \boldsymbol{\Gamma}_{0, i}^{(K)} \widehat{\boldsymbol{Z}}_{i}
\end{array}\right)^{\mathrm{T}}\left(\boldsymbol{C}_{n}^{0}\right)^{-1} \sum_{i=1}^{n}\left(\begin{array}{c}
\boldsymbol{D}_{i}^{\mathrm{T}} \boldsymbol{\Delta}_{0, i} \boldsymbol{V}_{0, i}^{(1)} \boldsymbol{e}_{i} \\
\vdots \\
\boldsymbol{D}_{i}^{\mathrm{T}} \boldsymbol{\Delta}_{0, i} \boldsymbol{V}_{0, i}^{(K)} \boldsymbol{e}_{i}
\end{array}\right) \\
& =\frac{1}{n^{2}} \sum_{1 \leq k, k^{\prime} \leq K}\left\{\sum_{i=1}^{n} \boldsymbol{D}_{i}^{\mathrm{T}} \boldsymbol{\Gamma}_{0, i}^{(k)} \widehat{\boldsymbol{Z}}_{i}\right\}^{\mathrm{T}} \boldsymbol{C}_{k k^{\prime}}^{0}\left\{\sum_{i=1}^{n} \boldsymbol{D}_{i}^{\mathrm{T}} \boldsymbol{\Delta}_{i} \boldsymbol{V}_{0, i}^{\left(k^{\prime}\right)} \boldsymbol{e}_{i}\right\} \\
& =\frac{1}{n^{2}} \sum_{1 \leq i, i^{\prime} \leq n} \widehat{\boldsymbol{Z}}_{i^{\prime}}^{\mathrm{T}}\left\{\sum_{1 \leq k, k^{\prime} \leq K} \boldsymbol{\Gamma}_{0, i^{\prime}}^{(k)} \boldsymbol{D}_{i^{\prime}} \boldsymbol{C}_{k k^{\prime}}^{0} \boldsymbol{D}_{i}^{\mathrm{T}} \boldsymbol{\Delta}_{0, i} \boldsymbol{V}_{0, i}^{\left(k^{\prime}\right)}\right\} \boldsymbol{e}_{i} .
\end{aligned}
$$

For any $\boldsymbol{c} \in \boldsymbol{R}^{d_{z}}$ and $\|\boldsymbol{c}\|=1$, we write

$$
n^{1 / 2} \boldsymbol{c}^{\mathrm{T}} \boldsymbol{A}_{n} \boldsymbol{\Sigma}_{n}^{-1 / 2} \boldsymbol{\Psi}_{n}^{-1}\left\{\dot{\boldsymbol{G}}_{\boldsymbol{\beta}}^{\mathrm{T}}\left(\widetilde{\boldsymbol{\theta}}_{0}\right)-\boldsymbol{H}_{\boldsymbol{\beta} \boldsymbol{\gamma}} \boldsymbol{H}_{\boldsymbol{\gamma} \boldsymbol{\gamma}}^{-1} \dot{\boldsymbol{G}}_{\boldsymbol{\gamma}}^{\mathrm{T}}\left(\widetilde{\boldsymbol{\theta}}_{0}\right)\right\}\left(\boldsymbol{C}_{n}^{0}\right)^{-1} \boldsymbol{G}_{n}^{0} \asymp \sum_{i=1}^{n} a_{i} \epsilon_{i},
$$

where

$$
a_{i}^{2}=n\left[\frac{1}{n} \sum_{i^{\prime}=1}^{n} \boldsymbol{c}^{\mathrm{T}} \boldsymbol{A}_{n} \boldsymbol{\Sigma}_{n}^{-1 / 2} \boldsymbol{\Psi}_{n}^{-1} \widehat{\boldsymbol{Z}}_{i^{\prime}}^{\mathrm{T}}\left\{\sum_{k, k^{\prime}=1}^{K} \boldsymbol{\Gamma}_{0, i^{\prime}}^{(k)} \boldsymbol{D}_{i^{\prime}} \boldsymbol{C}_{k k^{\prime}}^{0} \boldsymbol{D}_{i}^{\mathrm{T}} \boldsymbol{\Gamma}_{0, i}^{\left(k^{\prime}\right)}\right\}\right]_{\boldsymbol{\Sigma}_{i}}^{\otimes 2} .
$$

Note that conditioning on $(\mathbb{X}, \mathbb{Z}), \epsilon_{i}$ are independent, in addition, we have 


$$
\begin{aligned}
\max a_{i}^{2} & =O_{P}\left(\rho_{n}^{-5} d_{n}\right) \text { and } \\
\sum_{i=1}^{n} a_{i}^{2} & =\operatorname{Var}\left\{\sqrt{n} \boldsymbol{c}^{\mathrm{T}} \boldsymbol{A}_{n} \boldsymbol{\Sigma}_{n}^{-1 / 2} \boldsymbol{\Psi}_{n}^{-1} \widehat{\boldsymbol{J}}_{\mathrm{DZ}}^{\mathrm{T}}\left(\boldsymbol{C}_{n}^{0}\right)^{-1} \boldsymbol{G}_{n}^{0} \mid \mathbb{X}, \mathbb{Z}\right\} \\
& =\frac{1}{n} \sum_{i=1}^{n}\left\{\boldsymbol{c}^{\mathrm{T}} \boldsymbol{A}_{n} \boldsymbol{\Sigma}_{n}^{-1 / 2} \boldsymbol{\Psi}_{n}^{-1} \widehat{\boldsymbol{J}}_{\mathrm{DZ}}^{\mathrm{T}}\left(\boldsymbol{C}_{n}^{0}\right)^{-1}\left(\begin{array}{c}
\boldsymbol{D}_{i}^{\mathrm{T}} \boldsymbol{\Delta}_{0, i} \boldsymbol{V}_{0, i}^{(1)} \boldsymbol{\Sigma}_{i}^{1 / 2} \\
\vdots \\
\boldsymbol{D}_{i}^{\mathrm{T}} \boldsymbol{\Delta}_{0, i} \boldsymbol{V}_{0, i}^{(K)} \boldsymbol{\Sigma}_{i}^{1 / 2}
\end{array}\right)\right\}
\end{aligned}
$$

Thus, one has $\max _{i} a_{i}^{2} / \sum_{i=1}^{n} a_{i}^{2}=O_{P}\left(\rho_{n}^{-5} n^{-1} d_{n}\right)=o_{P}(1)$. By the LinderbergFeller central limit theorem, one has $\sum_{i=1}^{n} a_{i} \epsilon_{i} /\left(\sum_{i=1}^{n} a_{i}^{2}\right)^{1 / 2} \rightarrow N(0,1)$. The desired result follows from the Cramér-Wold device.

Similar to (A.2), we define

$$
\begin{aligned}
\widetilde{\boldsymbol{S}}_{n}(\boldsymbol{\beta}) & =\dot{\widetilde{\boldsymbol{G}}}_{n}^{\mathrm{T}}(\boldsymbol{\beta}) \widetilde{\boldsymbol{C}}_{n}^{-1}(\boldsymbol{\beta}) \widetilde{\boldsymbol{G}}_{n}(\boldsymbol{\beta}), \\
\widetilde{\boldsymbol{H}}_{n}(\boldsymbol{\beta}) & =\dot{\widetilde{\boldsymbol{G}}}_{n}^{\mathrm{T}}(\boldsymbol{\beta}) \widetilde{\boldsymbol{C}}_{n}^{-1}(\boldsymbol{\beta}) \dot{\widetilde{\boldsymbol{G}}}_{n}(\boldsymbol{\beta}) .
\end{aligned}
$$

Lemma S.13. Under Conditions (C2)-(C8), as $n \rightarrow \infty$,

$$
\sqrt{n} \widetilde{\boldsymbol{A}}_{n} \widetilde{\boldsymbol{\Sigma}}_{\mathrm{QIF}}^{-1 / 2}\left(\widetilde{\boldsymbol{\beta}}_{\mathrm{QIF}}-\boldsymbol{\beta}\right) \longrightarrow N\left(\mathbf{0}, \widetilde{\boldsymbol{\Sigma}}_{A}\right)
$$

where $\widetilde{\boldsymbol{A}}_{n}$ is any $q \times d_{z}$ matrix with a finite $q$ such that $\widetilde{\boldsymbol{A}}_{n}^{\otimes 2}$ converges to a nonnegative symmetric matrix $\widetilde{\mathbf{\Sigma}}_{A}$, and $\widetilde{\mathbf{\Sigma}}_{\mathrm{QIF}}=\lim _{n \rightarrow \infty} \mathbf{\Psi}_{n}^{-1} \boldsymbol{\Omega}_{n} \mathbf{\Psi}_{n}^{-1}$ with $\boldsymbol{\Psi}_{n}=\boldsymbol{J}_{\mathrm{DZ}}^{\mathrm{T}}\left(\boldsymbol{C}_{n}^{0}\right)^{-1} \boldsymbol{J}_{\mathrm{DZ}}$ and $\boldsymbol{\Omega}_{n}=\frac{1}{n} \sum_{i=1}^{n}\left\{\boldsymbol{J}_{\mathrm{DZ}}^{\mathrm{T}}\left(\boldsymbol{C}_{n}^{0}\right)^{-1} \boldsymbol{W}_{i}\right\}^{\otimes 2}$ for $\boldsymbol{W}_{i}$ in (2.8).

Proof. Let $\dot{\widetilde{Q}}_{n}$ and $\ddot{\widetilde{Q}}_{n}$ be the gradient vector and Hessian matrix of $\widetilde{Q}_{n}$. By Taylor's expansion,

$$
\begin{aligned}
\dot{\widetilde{Q}}_{n}\left(\widetilde{\boldsymbol{\beta}}_{\mathrm{QIF}}\right)-\dot{\widetilde{Q}}_{n}\left(\boldsymbol{\beta}_{0}\right)=\ddot{\widetilde{Q}}_{n}(\boldsymbol{\beta})\left(\widetilde{\boldsymbol{\beta}}_{\mathrm{QIF}}-\boldsymbol{\beta}_{0}\right) \\
\quad+\left.\frac{1}{2}\left(\widetilde{\boldsymbol{\beta}}^{\mathrm{QIF}}-\widetilde{\boldsymbol{\beta}}_{0}\right)^{\mathrm{T}} \frac{\partial \dot{\widetilde{Q}}_{n}(\boldsymbol{\beta})}{\partial \boldsymbol{\beta} \partial \boldsymbol{\beta}^{\mathrm{T}}}\right|_{\boldsymbol{\beta}=\boldsymbol{\beta}^{*}}\left(\widetilde{\boldsymbol{\beta}}^{\mathrm{QIF}}-\widetilde{\boldsymbol{\beta}}_{0}\right),
\end{aligned}
$$

where $\boldsymbol{\beta}^{*}=t \widetilde{\boldsymbol{\beta}}_{\mathrm{QIF}}+(1-t) \boldsymbol{\beta}_{0}$ for some $t \in[0,1]$. Note that $\dot{\widetilde{Q}}_{n}\left(\widetilde{\boldsymbol{\beta}}_{\mathrm{QIF}}\right)=0$ since $\widetilde{\boldsymbol{\beta}}_{\mathrm{QIF}}$ is the minimizer of $\widetilde{Q}_{n}(\boldsymbol{\beta})$, thus we have

$$
\begin{aligned}
-n^{-1} \dot{\widetilde{Q}}_{n}\left(\boldsymbol{\beta}_{0}\right)=n^{-1} & \ddot{\widetilde{Q}}_{n}\left(\boldsymbol{\beta}^{*}\right)\left(\widetilde{\boldsymbol{\beta}}_{\mathrm{QIF}}-\boldsymbol{\beta}_{0}\right) \\
& +\left.\frac{1}{2} n^{-1}\left(\widetilde{\boldsymbol{\beta}}_{\mathrm{QIF}}-\widetilde{\boldsymbol{\beta}}_{0}\right)^{\mathrm{T}} \frac{\partial \dot{\widetilde{Q}}_{n}(\boldsymbol{\beta})}{\partial \boldsymbol{\beta} \partial \boldsymbol{\beta}^{\mathrm{T}}}\right|_{\boldsymbol{\beta}=\boldsymbol{\beta}^{*}}\left(\widetilde{\boldsymbol{\beta}}_{\mathrm{QIF}}-\widetilde{\boldsymbol{\beta}}_{0}\right) .
\end{aligned}
$$


According to the Cauchy-Schwarz inequality, one has

$$
\left\|n^{-1}\left(\widetilde{\boldsymbol{\beta}}^{\mathrm{QIF}}-\widetilde{\boldsymbol{\beta}}_{0}\right)^{\mathrm{T}} \frac{\partial \dot{\widetilde{Q}}_{n}(\boldsymbol{\beta})}{\partial \boldsymbol{\beta} \partial \boldsymbol{\beta}^{\mathrm{T}}}\left(\widetilde{\boldsymbol{\beta}}_{\mathrm{QIF}}-\widetilde{\boldsymbol{\beta}}_{0}\right)\right\|^{2}=O_{P}\left(d_{n}^{2} / n^{2}\right) O_{p}\left(d_{n}^{3}\right)=o_{P}\left(n^{-1}\right) .
$$

By $[2]$,

$$
n^{-1} \dot{\widetilde{Q}}_{n}(\boldsymbol{\beta})=2 \widetilde{\boldsymbol{S}}_{n}(\boldsymbol{\beta})+O_{P}\left(n^{-1} \rho_{n}^{-1} d_{n}\right)=\mathbf{0}, n^{-1} \ddot{\widetilde{Q}}_{n}(\boldsymbol{\beta})=2 \widetilde{\boldsymbol{H}}_{n}(\boldsymbol{\beta})+o_{P}(1)
$$

where $\boldsymbol{S}_{n}(\boldsymbol{\beta})$ and $\boldsymbol{H}_{n}(\boldsymbol{\beta})$ are defined in (S.18) and (S.19). Thus,

$$
\begin{aligned}
-\left\{2 \widetilde{\boldsymbol{S}}_{n}\left(\boldsymbol{\beta}_{0}\right)+O_{P}\left(n^{-1} \rho_{n}^{-1} d_{n}\right)\right\}= & \left\{2 \widetilde{\boldsymbol{H}}_{n}\left(\boldsymbol{\beta}_{0}\right)+o_{P}(1)\right\}\left(\widetilde{\boldsymbol{\beta}}_{\mathrm{QIF}}-\boldsymbol{\beta}_{0}\right) \\
& +o_{P}\left(n^{-1 / 2}\right) .
\end{aligned}
$$

By Lemma S.10, the asymptotic distribution of $\sqrt{n} \widetilde{\boldsymbol{A}}_{n} \widetilde{\boldsymbol{\Sigma}}_{\mathrm{QIF}}^{-1 / 2}\left(\widetilde{\boldsymbol{\beta}}_{\mathrm{QIF}}-\boldsymbol{\beta}_{0}\right)$ is the same as the asymptotic distribution $\sqrt{n} \widetilde{\boldsymbol{A}}_{n} \widetilde{\boldsymbol{\Sigma}}_{\mathrm{QIF}}^{-1 / 2} \widetilde{\boldsymbol{H}}_{n}^{-1}\left(\boldsymbol{\beta}_{0}\right) \widetilde{\boldsymbol{S}}_{n}\left(\boldsymbol{\beta}_{0}\right)$. Similar to the proof of Lemma S.12, here we derive the asymptotic normality of $\sqrt{n} \widetilde{\boldsymbol{A}}_{n} \widetilde{\boldsymbol{\Sigma}}_{\mathrm{QIF}}^{-1 / 2} \widetilde{\boldsymbol{H}}_{n}^{-1}\left(\boldsymbol{\beta}_{0}\right) \widetilde{\boldsymbol{S}}_{n}\left(\boldsymbol{\beta}_{0}\right)$ by using the Cramér-Wold device and checking the Linderberg Condition. Note that $\widetilde{\boldsymbol{H}}_{n}\left(\boldsymbol{\beta}_{0}\right)=\boldsymbol{\Psi}_{n}+O_{P}\left(\sqrt{d_{n}^{3} / n}\right)$ by Lemma S.6, where $\boldsymbol{\Psi}_{n}=\boldsymbol{J}_{\mathrm{DZ}}^{\mathrm{T}}\left(\boldsymbol{C}_{n}^{0}\right)^{-1} \boldsymbol{J}_{\mathrm{DZ}}$, and

$$
\begin{aligned}
\sqrt{n} & \widetilde{\boldsymbol{A}}_{n} \widetilde{\boldsymbol{\Sigma}}_{\mathrm{QIF}}^{-1 / 2} \widetilde{\boldsymbol{H}}_{n}^{-1}\left(\boldsymbol{\beta}_{0}\right) \dot{\widetilde{\boldsymbol{G}}}_{n}^{\mathrm{T}}\left(\boldsymbol{\beta}_{0}\right) \widetilde{\boldsymbol{C}}_{n}^{-1}\left(\boldsymbol{\beta}_{0}\right) \widetilde{\boldsymbol{G}}_{n}\left(\boldsymbol{\beta}_{0}\right) \\
= & \sqrt{n} \widetilde{\boldsymbol{A}}_{n} \widetilde{\boldsymbol{\Sigma}}_{\mathrm{QIF}}^{-1 / 2} \widetilde{\boldsymbol{H}}_{n}^{-1}\left(\boldsymbol{\beta}_{0}\right) \dot{\widetilde{\boldsymbol{G}}}_{n}^{\mathrm{T}}\left(\boldsymbol{\beta}_{0}\right)\left(\boldsymbol{C}_{n}^{0}\right)^{-1} \widetilde{\boldsymbol{G}}_{n}\left(\boldsymbol{\beta}_{0}\right) \\
& +\sqrt{n} \widetilde{\boldsymbol{A}}_{n} \widetilde{\boldsymbol{\Sigma}}_{\mathrm{QIF}}^{-1 / 2} \widetilde{\boldsymbol{H}}_{n}^{-1}\left(\boldsymbol{\beta}_{0}\right) \dot{\widetilde{\boldsymbol{G}}}_{n}^{\mathrm{T}}\left(\boldsymbol{\beta}_{0}\right) \widetilde{\boldsymbol{C}}_{n}^{-1}\left(\boldsymbol{\beta}_{0}\right)\left\{\boldsymbol{C}_{n}^{0}-\widetilde{\boldsymbol{C}}_{n}\left(\boldsymbol{\beta}_{0}\right)\right\}\left(\boldsymbol{C}_{n}^{0}\right)^{-1} \widetilde{\boldsymbol{G}}_{n}\left(\boldsymbol{\beta}_{0}\right) \\
= & \sqrt{n} \widetilde{\boldsymbol{A}}_{n} \widetilde{\boldsymbol{\Sigma}}_{\mathrm{QIF}}^{-1 / 2} \widetilde{\boldsymbol{H}}_{n}^{-1}\left(\boldsymbol{\beta}_{0}\right)\left\{\boldsymbol{J}_{\mathrm{DZ}}+O_{P}\left(J_{n}^{1-r}\right)\right\}^{\mathrm{T}}\left\{\left(\boldsymbol{C}_{n}^{0}\right)^{-1}+o_{P}\left(\rho_{n}^{-2} d_{x} J_{n}^{r}\right)\right\} \\
& \times\left\{\widetilde{\boldsymbol{G}}_{n}^{0}+O_{P}\left(J_{n}^{-r}\right)\right\} \\
= & \sqrt{n} \widetilde{\boldsymbol{A}}_{n} \widetilde{\boldsymbol{\Sigma}}_{\mathrm{QIF}}^{-1 / 2} \boldsymbol{\Psi}_{n}\left\{\boldsymbol{J}_{\mathrm{DZ}}^{\mathrm{T}}\left(\boldsymbol{C}_{n}^{0}\right)^{-1} \widetilde{\boldsymbol{G}}_{n}^{0}\right\}\left\{1+o_{P}(1 / \sqrt{n})\right\} .
\end{aligned}
$$

For any $\boldsymbol{c} \in \boldsymbol{R}^{d_{z}}$ and $\|\boldsymbol{c}\|=1$,

$$
\begin{aligned}
\sqrt{n} \boldsymbol{c}^{\mathrm{T}} & \widetilde{\boldsymbol{A}}_{n} \widetilde{\boldsymbol{\Sigma}}_{\mathrm{QIF}}^{-1 / 2} \boldsymbol{\Psi}_{n}\left\{\boldsymbol{J}_{\mathrm{DZ}}^{\mathrm{T}}\left(\boldsymbol{C}_{n}^{0}\right)^{-1} \widetilde{\boldsymbol{G}}_{n}^{0}\right\} \\
= & \sqrt{n} \sum_{i=1}^{n} \boldsymbol{c}^{\mathrm{T}} \widetilde{\boldsymbol{A}}_{n} \widetilde{\boldsymbol{\Sigma}}_{\mathrm{QIF}}^{-1 / 2} \boldsymbol{\Psi}_{n}\left[\frac{1}{n} \sum_{i^{\prime}=1}^{n} \boldsymbol{Z}_{i^{\prime}}^{\mathrm{T}}\left\{\sum_{k, k^{\prime}=1}^{K} \boldsymbol{\Gamma}_{0, i^{\prime}}^{(k)} \boldsymbol{D}_{i^{\prime}} \boldsymbol{C}_{k k^{\prime}}^{0} \boldsymbol{D}_{i}^{\mathrm{T}} \boldsymbol{\Gamma}_{0, i}^{\left(k^{\prime}\right)}\right\}\right] \boldsymbol{e}_{i} \\
= & \sum_{i=1}^{n} b_{i} \epsilon_{i},
\end{aligned}
$$


where

$$
b_{i}^{2}=n\left[\frac{1}{n} \sum_{i^{\prime}=1}^{n} \boldsymbol{c}^{\mathrm{T}} \widetilde{\boldsymbol{A}}_{n} \widetilde{\boldsymbol{\Sigma}}_{\mathrm{QIF}}^{-1 / 2} \boldsymbol{\Psi}_{n} \boldsymbol{Z}_{i^{\prime}}^{\mathrm{T}}\left\{\sum_{k, k^{\prime}=1}^{K} \boldsymbol{\Gamma}_{0, i^{\prime}}^{(k)} \boldsymbol{D}_{i^{\prime}} \boldsymbol{C}_{k k^{\prime}}^{0} \boldsymbol{D}_{i}^{\mathrm{T}} \boldsymbol{\Gamma}_{0, i}^{\left(k^{\prime}\right)}\right\}\right]_{\boldsymbol{\Sigma}_{i}}^{\otimes 2} .
$$

Note that $\max b_{i}^{2}=O_{P}\left(\rho_{n}^{-5} d_{n}\right)$ and

$$
\begin{aligned}
\sum_{i=1}^{n} b_{i}^{2} & =\operatorname{Var}\left\{\sqrt{n} \boldsymbol{c}^{\mathrm{T}} \boldsymbol{A}_{n} \widetilde{\boldsymbol{\Sigma}}_{\mathrm{QIF}}^{-1 / 2} \boldsymbol{\Psi}_{n}\left\{\boldsymbol{J}_{\mathrm{DZ}}^{\mathrm{T}}\left(\boldsymbol{C}_{n}^{0}\right)^{-1} \widetilde{\boldsymbol{G}}_{n}^{0}\right\} \mid \mathbb{X}, \mathbb{Z}\right\} \\
& =\frac{1}{n} \sum_{i=1}^{n}\left\{\boldsymbol{c}^{\mathrm{T}} \widetilde{\boldsymbol{A}}_{n} \widetilde{\boldsymbol{\Sigma}}_{\mathrm{QIF}}^{-1 / 2} \boldsymbol{\Psi}_{n} \boldsymbol{J}_{\mathrm{DZ}}^{\mathrm{T}}\left(\boldsymbol{C}_{n}^{0}\right)^{-1} \boldsymbol{W}_{i}\right\}^{\otimes 2}
\end{aligned}
$$

Thus, one has $\max _{i} b_{i}^{2} / \sum_{i=1}^{n} b_{i}^{2}=O_{P}\left(\rho_{n}^{-5} n^{-1} d_{n}\right)=o_{P}(1)$. The LinderbergFeller central limit theorem implies that $\sum_{i=1}^{n} b_{i} \epsilon_{i} /\left(\sum_{i=1}^{n} b_{i}^{2}\right)^{1 / 2} \rightarrow N(0,1)$. The proof is complete.

\section{References.}

[1] He, X. and SHI, P. (1996). Bivariate tensor-product $B$-splines in a partly linear model. J. Multivariate Anal. 58 162-181. MR1405586

[2] QU, A., Lindsay, B. G. and Li, B. (2000). Improving generalised estimating equations using quadratic inference functions. Biometrika 87 823-836. MR1813977

[3] Stone, C. J. (1985). Additive regression and other nonparametric models. Ann. Statist. 13 689-705. MR0790566

[4] Wang, L., Liu, X., Liang, H. and Carroll, R. (2011). Estimation and variable selection for generalized additive partial linear models. Ann. Statist. 39 1827-1851. MR2893854

[5] Xue, L., Qu, A. and Zhou, J. (2010). Consistent model selection for marginal generalized additive model for correlated data. J. Amer. Statist. Assoc. 105 1518-1530. MR2796568

Li WANG

Department of Statistics

UNIVERSITY OF GEORGIA

Athens, GA 30602, USA

E-MAIL: lilywang@uga.edu

ANNiE QU

Department of Statistics

University of IlLinois at URbana-Champaign

Champaign, IL 61820, USA

E-MAIL: anniequ@illinois.edu
Lan Xue

Department of Statistics Oregon State University Corvallis, OR 97331, USA

E-MAIL: xuel@stat.oregonstate.edu

HUA LIANG

Department of Statistics

George Washington University WAShington, D.C. 20052, USA

E-MAIL: hliang@gwu.edu 